\title{
A THREE-DIMENSIONAL ANATOMICALLY ACCURATE FINITE ELEMENT MODEL FOR NERVE FIBER ACTIVATION SIMULATION COUPLING
}

\author{
A Thesis \\ presented to \\ the Faculty of California Polytechnic State University, \\ San Luis Obispo
}

\author{
In Partial Fulfillment \\ of the Requirements for the Degree \\ Master of Science in Biomedical Engineering
}

by

Shaina Ann Fischer

March 2015 
(C) 2015

Shaina Ann Fischer

ALL RIGHTS RESERVED 
TITLE:

AUTHOR:

DATE SUBMITTED:

COMMITTEE CHAIR:

COMMITTEE MEMBER:

COMMITTEE MEMBER:
A Three-Dimensional Anatomically Accurate Finite Element Model for Nerve Fiber Activation Simulation Coupling

Shaina Ann Fischer

March 2015

Robert Szlavik, Ph.D.

Professor of Biomedical Engineering

Scott Hazelwood, Ph.D.

Professor of Biomedical Engineering

Kristen Cardinal, Ph.D.

Professor of Biomedical Engineering 


\begin{abstract}
A Three-Dimensional Anatomically Accurate Finite Element Model for Nerve Fiber Activation Simulation Coupling

Shaina Ann Fischer

Improved knowledge of human nerve function and recruitment would enable innovation in the Biomedical Engineering field. Better understanding holds the potential for greater integration between devices and the nervous system as well as the ability to develop therapeutic devices to treat conditions affecting the nervous system.

This work presents a three-dimensional volume conductor model of the human arm for coupling with code describing nerve membrane characteristics. The model utilizes an inhomogeneous medium composed of bone, muscle, skin, nerve, artery, and vein. Dielectric properties of each tissue were collected from the literature and applied to corresponding material subdomains. Both a fully anatomical version and a simplified version are presented.
\end{abstract}

The computational model for this study was developed in COMSOL and formatted to be coupled with SPICE netlist code. Limitations to this model due to computational power as well as future work are discussed. The final model incorporated both anatomically correct geometries and simplified geometries to enhance computational power. A stationary study was performed implementing a boundary current source through the surface of a conventionally placed electrode. Results from the volume conductor study are presented and validated through previous studies.

Keywords: Finite element model, Volume conductor simulation, Myelinated neuron model 


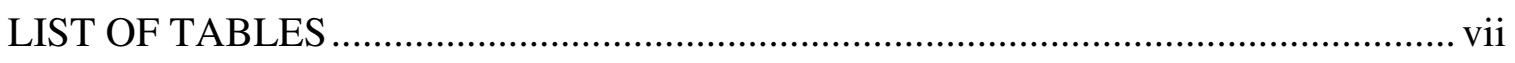

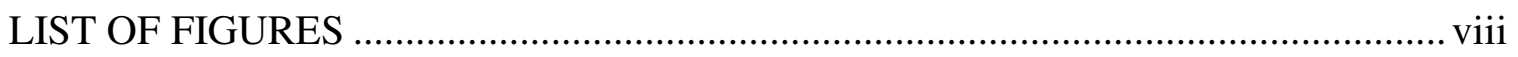
CHAPTER

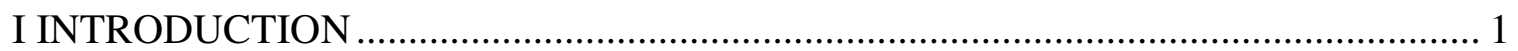

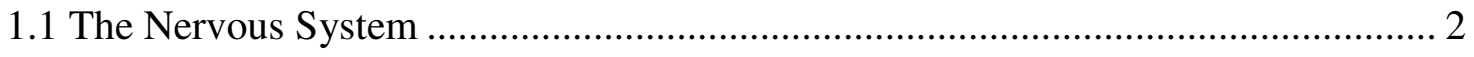

1.1.1 Physiology of the Nervous System............................................................. 2

1.1.2 Neuron Anatomy ...................................................................................... 3

1.1.3 Membrane Potential and Impulse Propagation................................................ 5

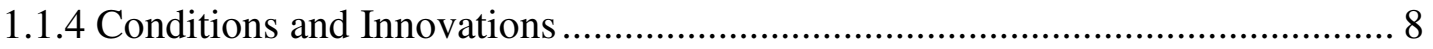

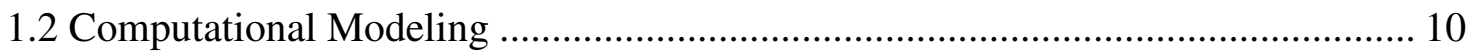

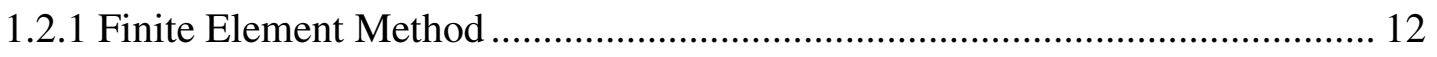

1.2.2 Significance of FEA in the Biomedical Field............................................ 14

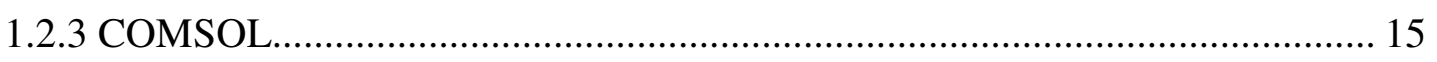

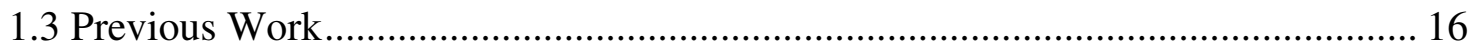

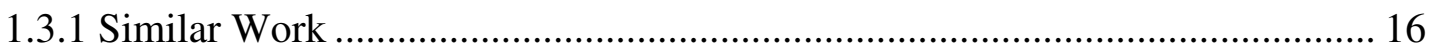

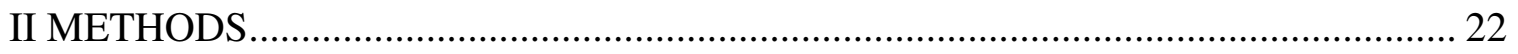

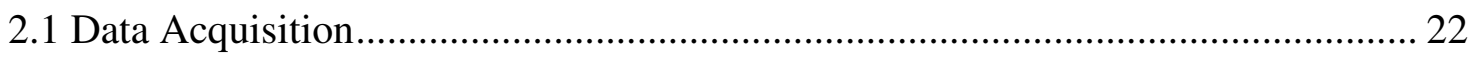

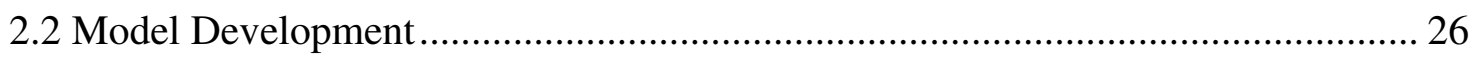

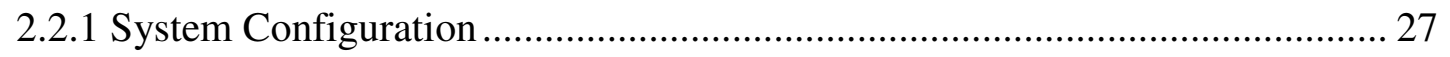

2.2.2 Governing Equations \& Constitutive Laws............................................... 33

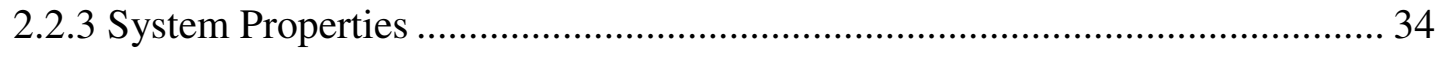

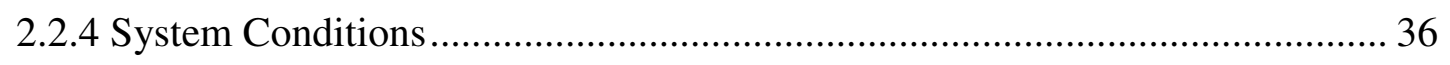

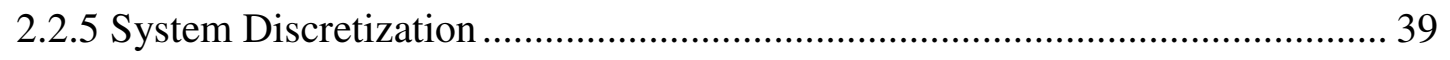

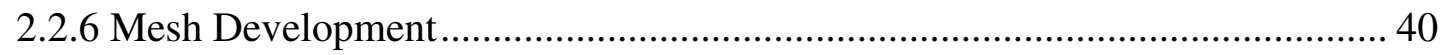

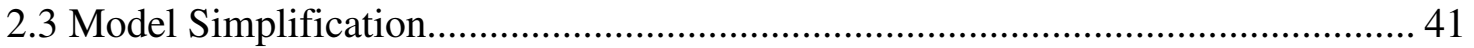

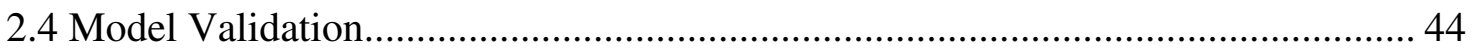

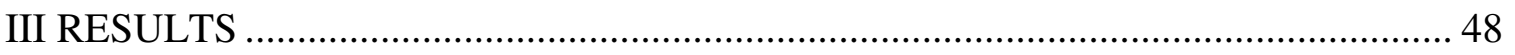




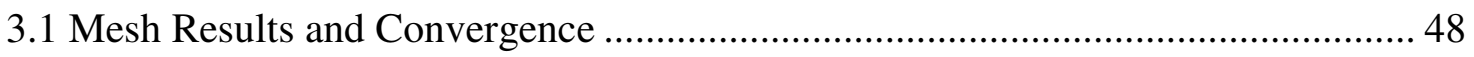

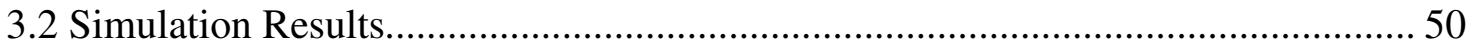

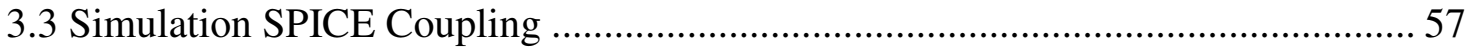

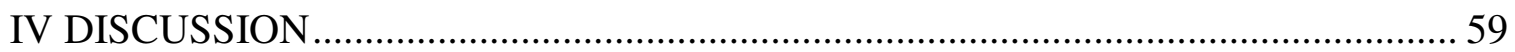

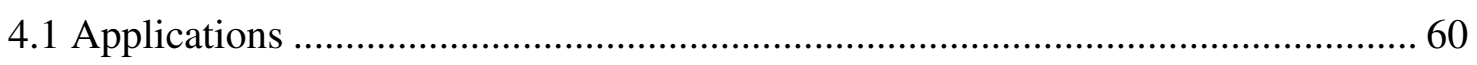

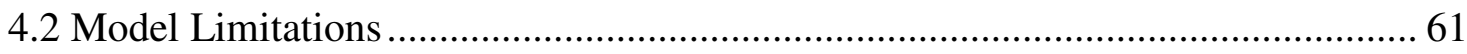

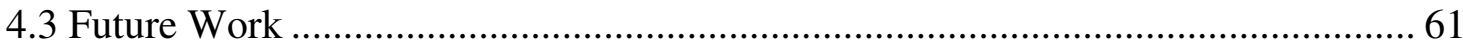

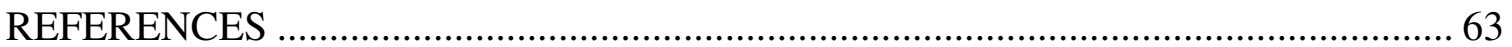




\section{LIST OF TABLES}

Table

Page

1. Summary of the dielectric properties applied for each tissue in COMSOL including sources for each property........................................................................ 36

2. Dielectric properties for each tissue used in the validation study and applied in COMSOL. 


\section{LIST OF FIGURES}

Figure

Page

1. Schematic representing the division of the nervous system in the central and

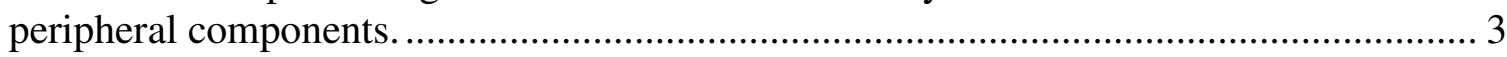

2. Anatomy of a typical motor neuron, representing dendrites, axon, and myelin sheath [2]......

3. The five steps of an action potential: (1) resting state, (2) depolarization, (3) repolarization, (4) hyperpolarization, (5) restored to resting membrane potential [4]. ..... 6

4. Illustration of the Nodes of Ranvier, where the electrical signal is allowed to jump from nodes along the axon [3].

5. The six step process for developing a computational model, with assumptions

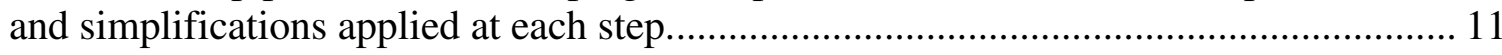

6. The finite element method, derived from a physical problem [12] ........................... 13

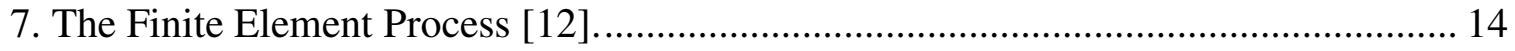

8. Homogenous isotropic model developed in COMSOL by Soto [17] ......................... 18

9. Non-homogenous isotropic model developed in COMSOL by Soto [17]................... 18

10. Two-dimensional geometry developed in COMSOL representing the human

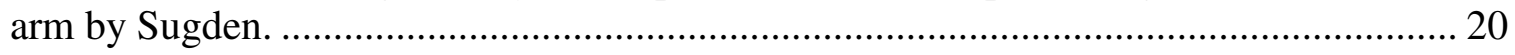

11. Human nerve membrane potential profile developed by Sugden............................ 20

12. A screenshot of the BodyParts3D online database, which allows the user to select individual objects or organize by systems [20]. 23

13. Raw file of the right humerus bone obtained from BodyParts3D in Wavefront OBJ format 24

14. Initial mesh generated on the right humerus bone in Meshlab. 25

15. The right humerus bone in netfabb Basic during mesh editing. 26

16. The six steps to developing a computational model, as in this work. 27

17. Complete assembly of the bones of the human arm, ready for import into COMSOL 
18. Complete assembly of the muscles of the human arm ready for import into

19. Complete assembly of the artery and vein of the human arm ready for import

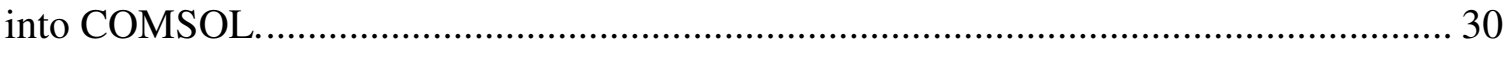

20. Final skin geometry, ready for import into COMSOL. ......................................... 30

21. Final geometry composition including bone, muscle, skin, artery, and vein with transparency enabled to enhance viewing.

22. Final geometry composition including bone, muscle, artery, and vein with skin hidden from view to enhance viewing.

23. Electric insulation boundary condition applied at all external surfaces of the arm, represented in purple

24. Ground boundary condition applied at the cross-section of the human arm (mid-bicep), represented in purple.

25. Current source boundary condition applied at the contact between skin and electrode, represented in purple.

26. Complete assembly of the human arm, pre-meshed in Meshlab and imported into COMSOL

27. Organization of muscle into complex fibrous groupings [27].

28. Enlarged view of simplified muscle structures, sectioned from bone to compose the simplified model.

29. Two-dimensional volume field conductor profile developed by Sugden. 46

30. Three-dimensional volume conductor profile developed in this study using equivalent properties for comparison with Figure 29.

31. Mesh convergence plot

32. Final mesh used to perform analysis in COMSOL.

33. Electric potential plot of the final volume field conductor simplified model. 51

34. The first cut plane, corresponding to the surface plot of Figure 35. 53

35. Electric potential surface plot of a slice taken from the volume conductor model. 53

36. The second cut plane, corresponding to the surface plot of Figure 37. 54 
37. Electric potential surface plot of a slice taken from the volume conductor

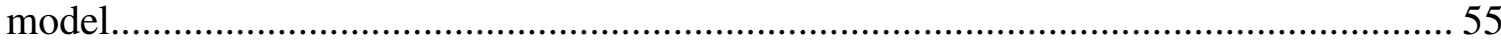

38. The third cut plane, corresponding to the surface plot of Figure 39.......................... 56

39. Electric potential surface plot of a slice taken from the volume conductor model..... 57 


\section{INTRODUCTION}

The nervous system and associated nerve cells are vital to health and well-being of human life. Nerve cells conduct impulses along their processes in order to transmit signals throughout the entire body. Signal transmission enables the function of each organ system, as well as somatic and autonomic movements and responses [1]. Better understanding of nerve cells and their behavior would facilitate innovation within the Biomedical Engineering field. Application of this understanding includes enhanced prosthetic integration, the ability for brain-machine interfacing, therapeutic devices for nervous system disorders, and pain management.

The aim of this work is to contribute to the understanding of human nerve function by creating a computational model of the human arm to be coupled with neural recruitment and excitation code. The model presented in this work incorporates precise anatomy and representative tissue properties into commercially available Finite Element Analysis software. Previous studies successfully created simplified two-dimensional models for coupling. This work attempts to prove the significance of a three-dimensional, anatomically accurate non-homogenous volume conductor model by comparison of analogous study results.

To support the understanding of the model defined in this work, the following are presented: an overview of the anatomy and physiology of the human nervous system and nerve cells, relevant conditions and disorders, the significance and implementation of computational modelling, and a review of previous work contributing to this study. 


\subsection{The Nervous System}

The nervous system is the target of many research efforts and innovations in the biomedical engineering field. Diseases and neuropathies associated with the nervous system are difficult to treat and can be detrimental to health and quality of life. Having a better understanding of neuron function and targeting capabilities would facilitate improved development of devices and therapies.

\subsubsection{Physiology of the Nervous System}

The human nervous system is comprised of two main components: the central nervous system (CNS) and the peripheral nervous system (PNS). The CNS consists of the brain and spinal cord, while the PNS consists of motor and sensory neurons. The role of the CNS is to interpret sensory information and send signals to the PNS. The PNS collects sensory information and delivers signals to muscles and glands. The motor neurons of the PNS can be further divided into somatic and autonomic components. The somatic component of the nervous system is responsible for voluntary movements and conducts impulses from the CNS to skeletal muscles, while the autonomic component of the nervous system is responsible for involuntary responses and conducts impulses from the CNS to cardiac muscle, smooth muscle, and glands. Furthermore, the autonomic nervous system is divided into sympathetic and parasympathetic divisions. The sympathetic division mobilizes body systems during activity; conversely the parasympathetic division conserves energy and promotes digestive functions during rest [1]. Figure 1 illustrates the divisions and functions of the nervous system. In essence the 
nervous system is responsible for every movement, feeling, and sense that the human body experiences.

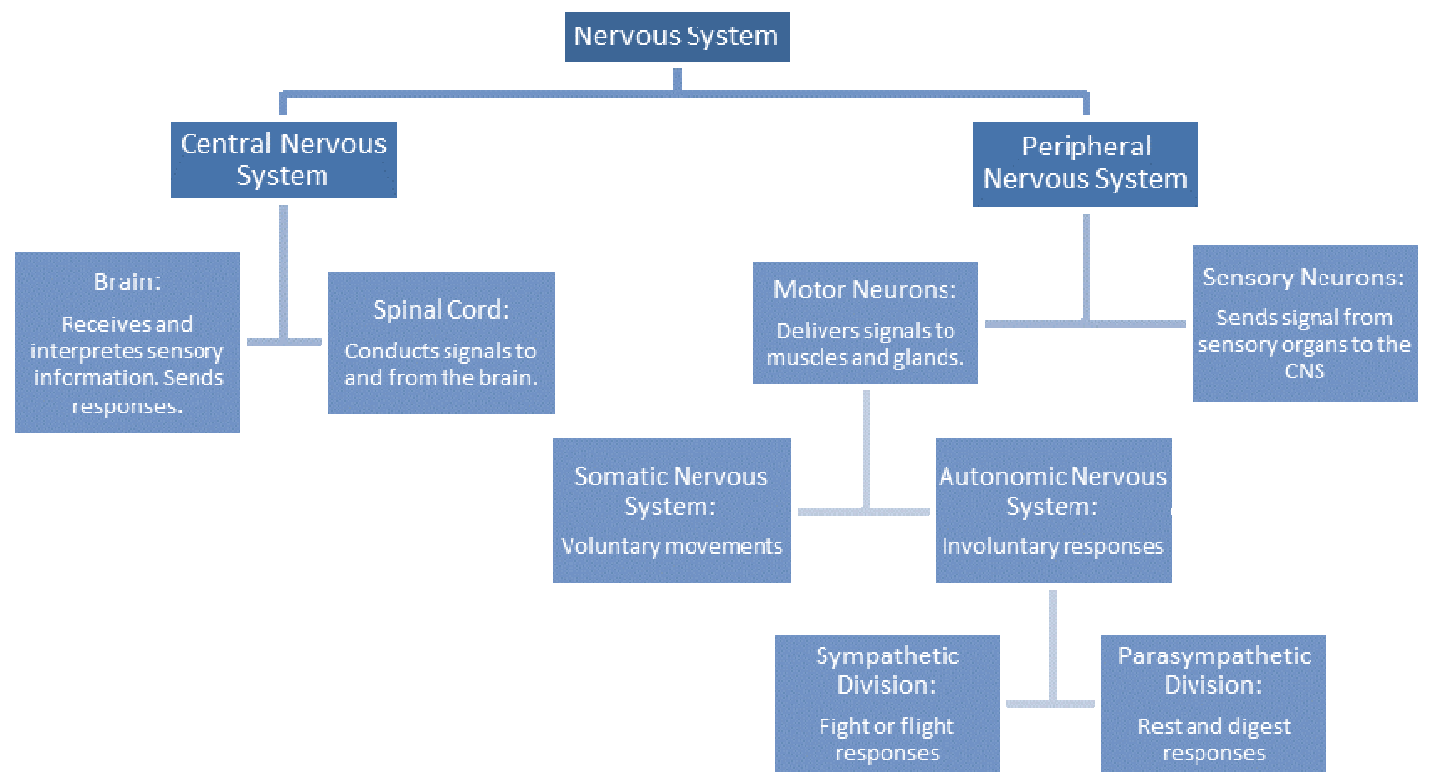

Figure 1: Schematic representing the division of the nervous system in the central and peripheral components.

\subsubsection{Neuron Anatomy}

Neurons are the individual nerve cells within the nervous system. They are highly specialized cells that conduct nerve impulses throughout the body. Neurons are unique cells in several ways. They have a distinctly long lifespan; neurons can function optimally for a lifetime [1]. They have a very high metabolic rate and require a continuous supply of oxygen and glucose to survive [1].

There are multiple types of neurons, each class with unique morphology and function. These large complex cells consist of a cell body and slender processes. Figure 2 illustrates an example of a motor neuron. There are two types of processes: dendrites and axons. The dendrites are short branches with a large surface area to receive signals and 
convey them toward the cell body [1]. The axon of each neuron can be long, reaching up to a meter in length. The axon conducts impulses away from the cell body, ending at the axon terminal where it releases a chemical signal of neurotransmitters to either excite or inhibit other neurons [1]. The three main types of neurons are sensory neurons, motor neurons, and interneurons. Sensory neurons are responsible for conducting sensory information from receptors to the central nervous system [1]. Motor neurons are responsible for conducting impulses from the central nervous system to a muscle or gland. Interneurons are neurons only found in the central nervous system and link sensory neurons with motor neurons [1]. Structurally, neurons can be classified into unipolar, bipolar, and multipolar. Unipolar neurons are typically sensory neurons with receptors in skin, muscle, or organs. They possess a single process with a long axon terminating in the spinal cord. Bipolar neurons possess two processes branching from the cell body [1]. Bipolar neurons are typically sensory neurons serving the visual and auditory systems and are short in length. Multipolar neurons possess multiple branches leaving the cell body to enhance integration. They typically have long axons, capable of reaching distant portions of the nervous system [1]. 


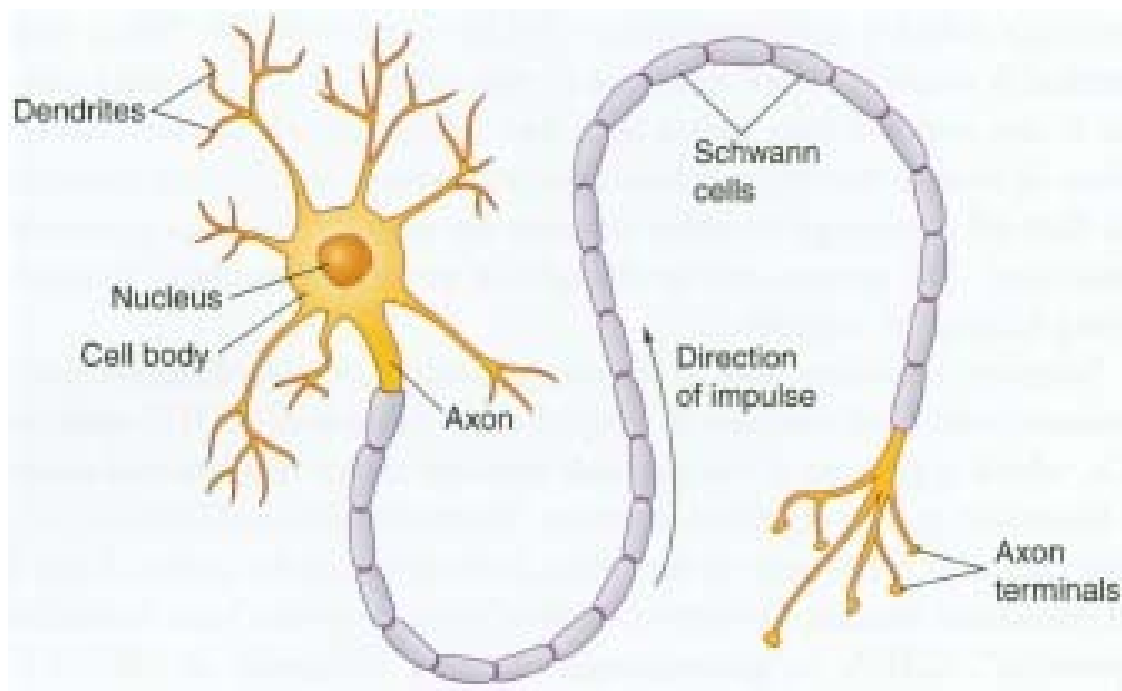

Figure 2: Anatomy of a typical motor neuron, representing dendrites, axon, and myelin sheath [2].

The axons of long nerve fibers are covered by a segmented myelin sheath. The myelin sheath is responsible for protecting and electrically insulating fibers as well as increasing the speed of transmitting nerve impulses. The myelin sheath in the PNS is formed by Schwann cells. Schwann cells wrap around the axon at regular intervals, leaving gaps called the nodes of Ranvier. These gaps provide a break in the insulation that allows impulses to jump and ultimately speeds transmission.

\subsubsection{Membrane Potential and Impulse Propagation}

Action potentials form the basis of signal transmission within neurons. Opening and closing of voltage-gated ion channels can cause a reversal of a neuron's resting membrane potential and generates an action potential [3]. Action potentials consists of five steps (Figure 3). Step one corresponds to resting state, during which no ions move through the voltage-gated channels. Step two corresponds to depolarization, where sodium ions flow into the cell. In step three, potassium ions flow out of the cell causing repolarization. In step four, the membrane is hyperpolarized because potassium ions 
continue to leave the cell. In this step the membrane is refractory and cannot generate a new action potential. Finally in step five, potassium channels close.

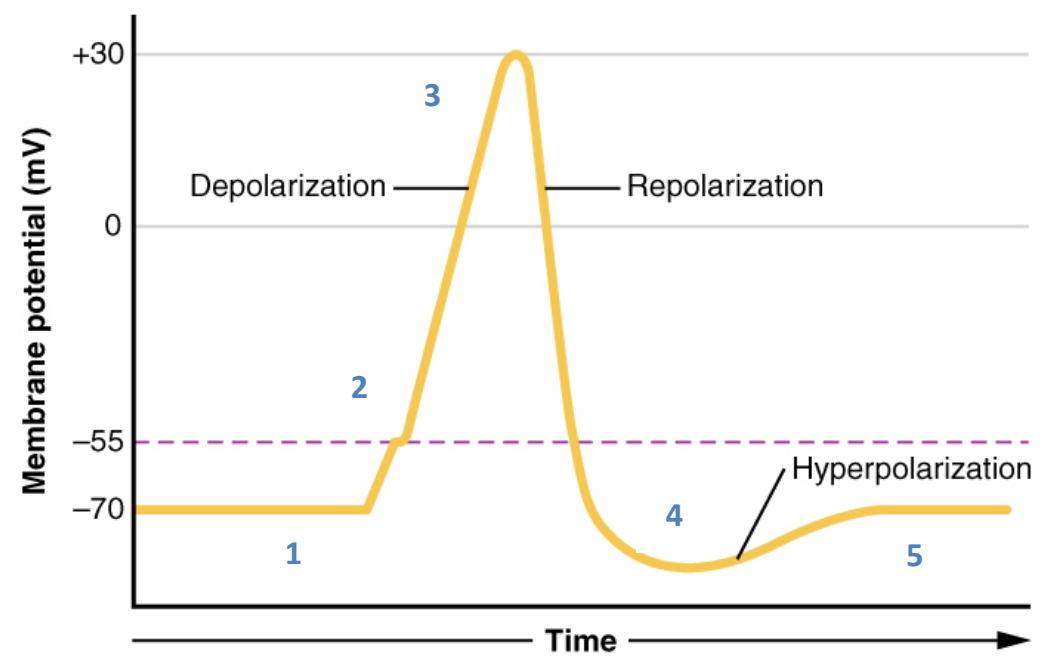

Figure 3: The five steps of an action potential: (1) resting state, (2) depolarization, (3) repolarization, (4) hyperpolarization, (5) restored to resting membrane potential [4].

Neurotransmitters released by axon terminals bind to receptors on another neuron's dendrites. In excitatory synapses, this causes ligand-gated ion channels to open and positive ions flow into the neuron and depolarize the membrane. Neurotransmitter release at inhibitory synapses causes hyperpolarization of the membrane [5].

Depolarization reduces the voltage drop between the inside and outside of the cell. Sensory or other stimuli depolarize the neuron to threshold potential (approximately $55 \mathrm{mV})$. Sodium channels at the beginning of the axon open to begin the action potential [3]. When depolarization is complete, the cell's membrane voltage returns to resting potential. The refractory period is initiated by the close of sodium channels. Voltagegated potassium channels open and allow potassium to leave the cell, returning membrane potential to a negative voltage. As potassium ions continue to diffuse out of 
the cell, it becomes hyperpolarized and causes the membrane potential to become more negative [3].

Conduction velocity of action potentials along the axon of a neuron varies widely. In neural pathways, nerve fibers can transmit impulses at a velocity of $100 \mathrm{~m} / \mathrm{s}$ or more. Slower conduction velocities are observed in internal organs such as the gut and blood vessels [1]. Impulse propagation is greatly dependent on two factors: axon diameter and myelination. Axon diameters vary between neurons; typically larger diameters allows faster conduction due to lowered resistance to current flow [1]. As an action potential travels down the axon, it is regenerated by voltage gated channels within the Nodes of Ranvier. If an axon has no myelination, the uninsulated space spans the entire length and ion channels are immediately adjacent to each other. This type of propagation is known as continuous conduction and is very slow [1]. Myelin insulates the axon to prevent leakage of charge and allows the membrane voltage to change more rapidly [1]. Because current can only pass through the membrane at the Nodes of Ranvier, the electrical signal jumps from node to node along the axon (Figure 4). This type of conduction is called saltatory conduction and is approximately thirty times faster than continuous conduction [1].

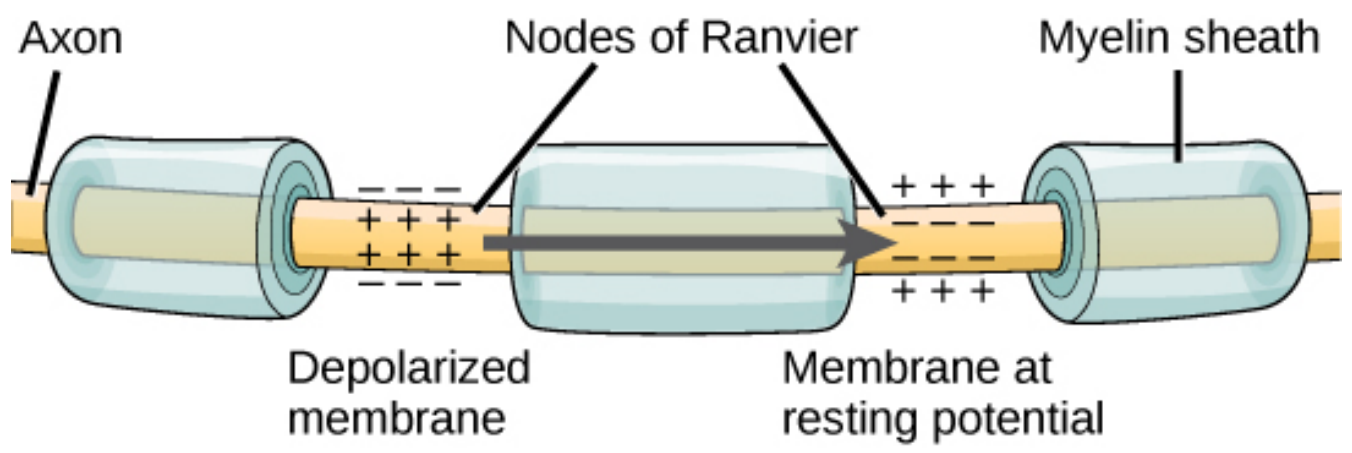

Figure 4: Illustration of the Nodes of Ranvier, where the electrical signal is allowed to jump from nodes along the axon [3]. 


\subsubsection{Conditions and Innovations}

There are many diseases associated with the nervous system. Currently, little is known or understood about many of these diseases. Understanding the function and behavior of neurons and the nervous system will improve scientists' abilities to develop cures and treatments.

Multiple sclerosis causes the degeneration of myelin [3]. This slows the conduction of action potentials by reducing the efficacy of saltatory conduction. Multiple sclerosis is an abnormal immune system response producing inflammation. As it damages and destroys myelin, it also causes damage to the nerve fiber. Symptoms of multiple sclerosis include cognitive difficulty, loss of balance, numbness, weakness, muscle spasms, and speech problems [6]. Treatment for multiple sclerosis is typically limited to symptom management. Scientists are currently investigating strategies to stimulate the repair and protection of myelin [7].

Another condition that affects the nervous system is peripheral neuropathy, a disorder of the peripheral nerves. It results from damage to the nerves causing impairment of sensation, movement, and organ function. There are many forms of peripheral neuropathy, each with its own characteristics and prognosis. Thirty percent of peripheral neuropathies remain idiopathic, however known causes include diabetes, autoimmune disease, tumor, toxins, and infection [8]. Some symptoms of peripheral neuropathy include numbness, tingling, pain, tremors, cramping, and sensory or motor loss [8]. Currently treatment for peripheral neuropathy is mainly symptomatic and lacks restorative or preventative therapy options. 
Another category of conditions that affect the nervous system are called degenerative nerve diseases. These diseases affect a wide range of body functions including balance, movement, speech, and organ function [9]. Causes of these diseases are often unknown but can include toxin and chemical exposure, virus, stroke, or genetics [9]. Degenerative nerve diseases include Alzheimer's disease, Huntington's disease, Spinal muscular atrophy, and Friedreich's Ataxia [9]. All of these diseases are debilitating or life-threatening. No cure is currently available, and treatment is only symptomatic to prolong and improve quality of life.

Currently, neural engineers are conducting studies to enable treatment of these conditions and manage associated pain. The conditions described in this work represent only a fraction of those affecting the nervous system. Greater knowledge of the nervous system and neuronal behavior will enhance the ability to prevent, treat, and manage conditions. Computational modeling is a way to study the behavior and characteristics of the nervous system.

Some neuroscience efforts are attempting to model neural systems to enhance understanding, interface, and manipulation of the nervous system [10]. Other efforts focus on neural signals and utilize quantitative techniques to measure and analyze the nature of neural communication [10]. One example of a significant innovation in the field of neural engineering is the bionic arm, an artificial limb for amputees enabling control of the device by the nervous system through the spinal cord [10]. The DEKA bionic arm was funded by the Defense Advanced Research Projects Agency (DARPA) involving a team of over three-hundred scientists and engineers led by Dean Kamen [10]. The DEKA bionic arm contains electrodes capable of detecting electrical impulses fired by the brain 
[10]. Additional functionality is provided by buttons built into the patient's shoes [10]. Other innovations utilizing the understanding of neuron function include pacemakers and neurostimulation systems to monitor and inhibit seizures. Potential innovations not yet achieved include pain inhibition, highly localized and reversible anesthesia, restoration of function to paralysis victims, and an artificial retina [10].

\subsection{Computational Modeling}

Computational modeling combines mathematics, physics, and computer science to study the behaviors and reactions of complex problems in-silico. A computational model contains many variables that characterize the system being studied [11]. The National Institutes of Health states that "modeling can expedite research by allowing scientists to conduct thousands of simulated experiments by computer in order to identify the actual physical experiments that are most likely to help the researcher find the solution to the problem being solved" [11]. When studying a complex problem, many different variables are involved. Computational modeling allows a researcher to change one variable and predict the significance and magnitude of its effect on the problem. This approach is extremely useful in research because it helps narrow down a problem, isolate significant factors, and validate the need for non in-silico tests. Computational modeling is also useful in device design because it can predict efficacy, illuminate unexpected behavior, validate expected behavior, and serve as an accurate test environment without the cost and time of bench and in-vivo testing. Optimal design and research practices incorporate computational models with experiments and in-vivo tests. These tests can be used to validate in-silico models and can provide evidence to support results determined from adjusted and more complex models. 
To develop a successful computational model, several steps must be considered in order to narrow down the scope, goal, and results of the model. Following the six step process outlined in Figure 5 will ensure that a model is relevant to the problem or situation. A complete computational model first considers the targeted system configuration. This includes the entire structure of the model, the geometries being used, and the scope of the situation. It is important to consider what portion of the problem is necessary to create a useful model. Second, the governing equations and constitutive laws that control the situation must be considered. Third, the system properties should be described. This involves any physical, biological, chemical, or other property that affects the system. Next, system conditions should be determined; this includes boundary conditions, applied conditions such as loadings, and initial conditions. This step is followed by system discretization, in which mesh refinement, mesh convergence, tolerances, and mathematical error should be considered. Finally, validation methods to prove accuracy of the model need to be determined. Validation can include previous studies, bench testing, in-vivo test results, and other experimentation.

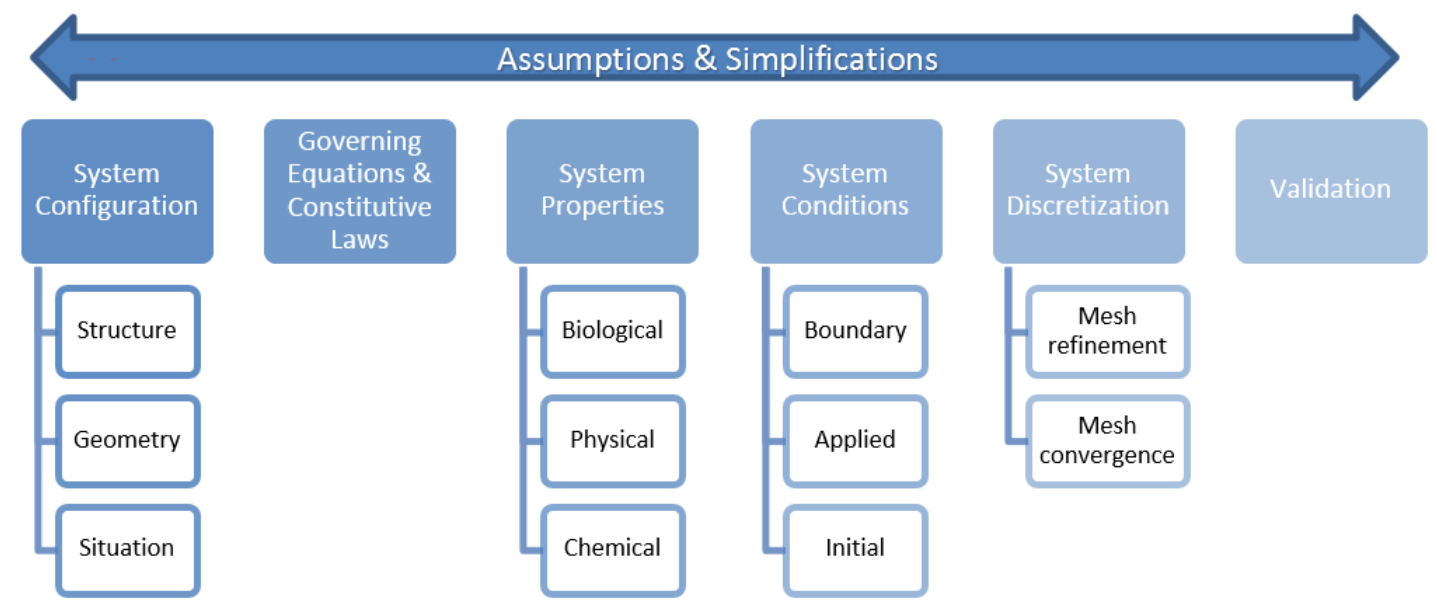

Figure 5: The six step process for developing a computational model, with assumptions and simplifications applied at each step. 
For each of step, assumptions and simplifications must be considered. This is an important component of model development. A computational model cannot always describe an entire system. The human body is a particularly complex system. To model a medical device or function of the body, simplifications and assumptions must be made in order to not exceed computational capabilities. The researcher must determine what degree of simplification will maximize computational cost and time without sacrificing accuracy.

\subsubsection{Finite Element Method}

Computational modelling incorporates the Finite Element Method, also known as Finite Element Analysis (FEA). The Finite Element Method is a "sophisticated numerical scheme used to approximate the solution to common boundary value problems" [12]. In FEA, the user makes informed decisions to set up a problem in order to develop a solution. Using FEA makes solving difficult problems possible by transferring facilitating computation by computer. To mathematically model a physical system, two different approaches can be taken to reach a solution. First, assumptions may be made to develop a simple model, and then an exact solution can be solved for. Second, a complex model incorporating important details may be developed, and an approximate solution can be solved for. This process is illustrated in Figure 6 [12]. The finite element method develops an approximate solution for an exact model. In this study, an exact model of the human arm was created in order to solve for an approximate solution. 


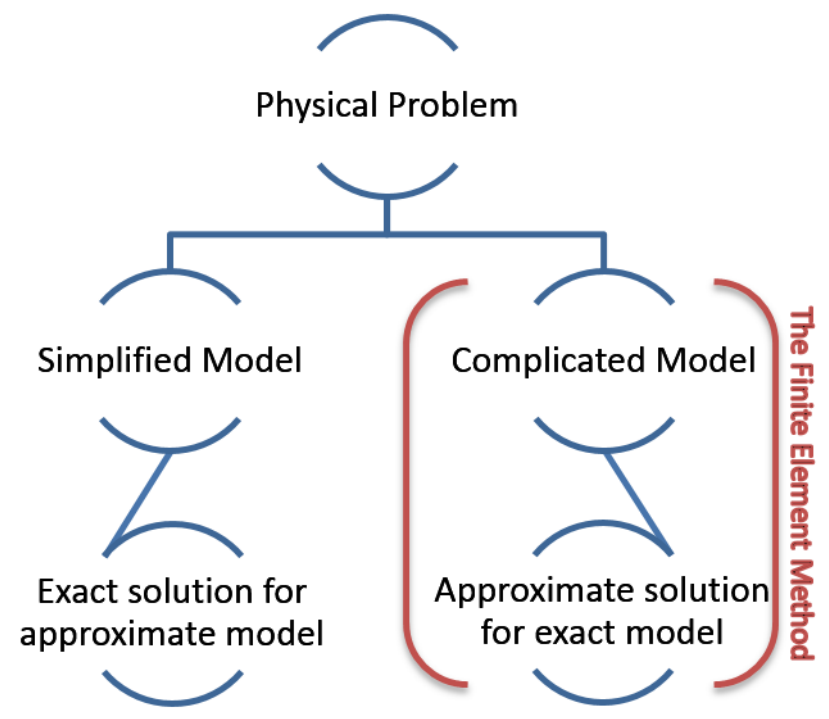

Figure 6: The finite element method, derived from a physical problem [12].

The Finite Element Process describes how a problem is translated into an FEA model, and then solved [12]. In the first step, the problem is defined. This step specifies what question you will attempt to answer with your FEA solution. In the second step, the model is defined. This step describes the scope, geometry, boundary conditions, loadings, and properties that go into the model. The third step is to mesh the model. Fourth, the analysis method must be chosen. In the fifth step, the FEA model is solved using commercial or custom code. Finally in the sixth step, post processing is performed to obtain results. Steps three through six encompass an iterative process, which can be followed to ensure that the FEA model achieves optimal results. This process is outlined in Figure 7 below. 


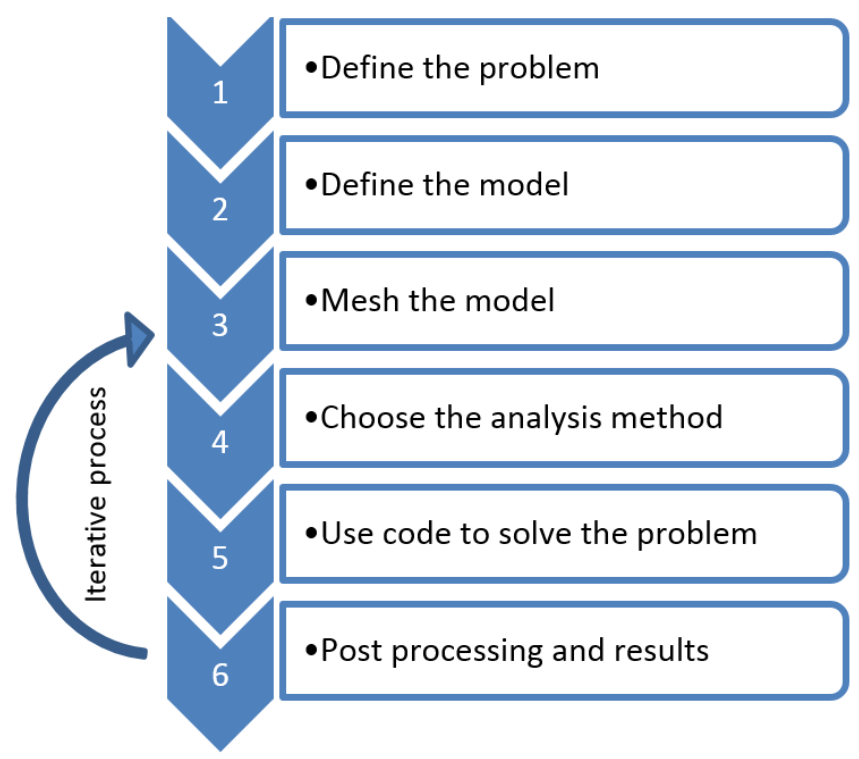

Figure 7: The Finite Element Process [12].

FEA uses matrix algebra on a mass scale to solve complex systems of equations at many points. These points are known as nodes within a mesh. A mesh is a system of geometric entities that covers the surface or volume of a model. Mesh elements come in different shapes, including triangles and rectangles. Various element types are used for different computations.

\subsubsection{Significance of FEA in the Biomedical Field}

The use of computational modeling and FEA in the biomedical engineering field has been growing in popularity as a tool to aid design, testing, and validation. In January of 2014, the Food and Drug Administration (FDA) Center for Devices and Radiologic Health (CDRH) issued a Guidance Document titled, "Reporting of Computational Modeling Studies in Medical Device Submissions" [13]. The purpose of this document is to provide instruction on proper use of computational modeling for device regulation. This document describes good practices in model development, as previously discussed 
and outlined in Figure 5. The FDA states: "computational modeling and simulation studies, together with bench, non-clinical in vivo, and clinical studies are tools that can be used to evaluate the safety and effectiveness of medical devices" [13]. With this Guidance Document, the FDA acknowledges the importance and impact of computational modelling in the biomedical industry.

\subsubsection{COMSOL}

Many commercially available programs exist today incorporating FEA and computational modeling with a graphical user-interface. One such available software platform is COMSOL. Founded in Stockholm, Sweden, the first multiphysics edition was released in 1998 [14]. The current software has capabilities in structural mechanics, electromagnetics, fluid flow, heat transfer, chemical reactions, acoustics, electric currents, and more [14]. Developing a model using this software allows the user to add various physics modules and couple them into a single simulation. The integrated work environment and user-interface allows for unified modeling workflow [14]. COMSOL features tools to draw geometries or import a CAD model, apply material properties, implement boundary conditions, incorporate user-defined variables and functions, generate a mesh, run stationary or time dependent simulations, and post-process. More advanced capabilities include linking multiple physics into one simulation, customizing studies by adding multiple steps to target results within specific time frames, generating adaptive meshes to adjust refinement as a study progresses, and Live-Link to other programs such as SolidWorks, MATLAB, and Excel to optimize cross-platform studies. 


\subsection{Previous Work}

Nerve fiber activation has been a major area of study for decades. In 1959 George Bishop published an article in the Journal of Nervous and Mental Disease titled "The Relation Between Nerve Fiber Size and Sensory Modality” [15]. Bishop proposed a relation between afferent nerve fiber size and physiology of sensory systems. Related work has since been aimed at understanding how stimuli levels vary depending upon characteristics of the nerve fiber. Better understanding of the stimulation of nerve fibers will provide insight to aid in the diagnosis and treatment of neuropathies.

In a study by Robert Szlavik and Hubert de Bruin titled "The effect of stimulus current pulse width on nerve fiber size recruitment patterns," a simulation was performed to study recruitment patterns within motor nerve fibers [16]. This simulation utilized accurate fiber diameters with skeletal tissue properties but did not include realistic inhomogeneous tissue layers [16]. Implementing a time varying field simulation coupled with nerve fiber excitation simulations, it was found that only a marginal selectivity is achievable [16]. Additionally Szlavik concluded that selectivity increases with increased electrode distance [16].

Studies like that performed by Bishop and Szlavik form the basis of nerve fiber activation simulation studies. Additional works relevant to the field and forming the basis for this study will now be discussed in further detail.

\subsubsection{Similar Work}

One previous study relevant to the investigation of nerve fiber activation was conducted by Nathan Soto under the direction of Dr. Robert Szlavik [17]. Published in 
2011 and titled "Characterizing Nerve Fiber Activation by Varying Fiber Diameter and Depth Within a Conductive Medium: A Finite Element Approach," Soto coupled MATLAB and COMSOL to develop a simulation to determine what stimuli were necessary to activate fibers of varying diameter and depth. COMSOL was used to describe the voltage profile present within a conductive medium environment of the human forearm after a stimulus is applied [17]. MATLAB was used to analyze COMSOL output and determine the activation status of a nerve fiber. Soto developed two separate but similar models in COMSOL; one using homogenous isoptropic material to represent the human forearm and the other inhomogeneous material [17]. Both models utilized a two-dimensional basic geometry with an applied constant DC source [17]. Below is the plot of the first isotropic, homogenous model (Figure 8). This volume plot demonstrates a maximum electric potential of $0.00227 \mathrm{~V}$. The second COMSOL model incorporated the nerve fiber as well as its dielectric properties. Several iterations of this study were performed to analyze various fiber characteristics. A profile plot from the second nonhomogeneous isotropic model can be found in Figure 9 below. This model demonstrates a maximum potential of $0.00202 \mathrm{~V}$. 


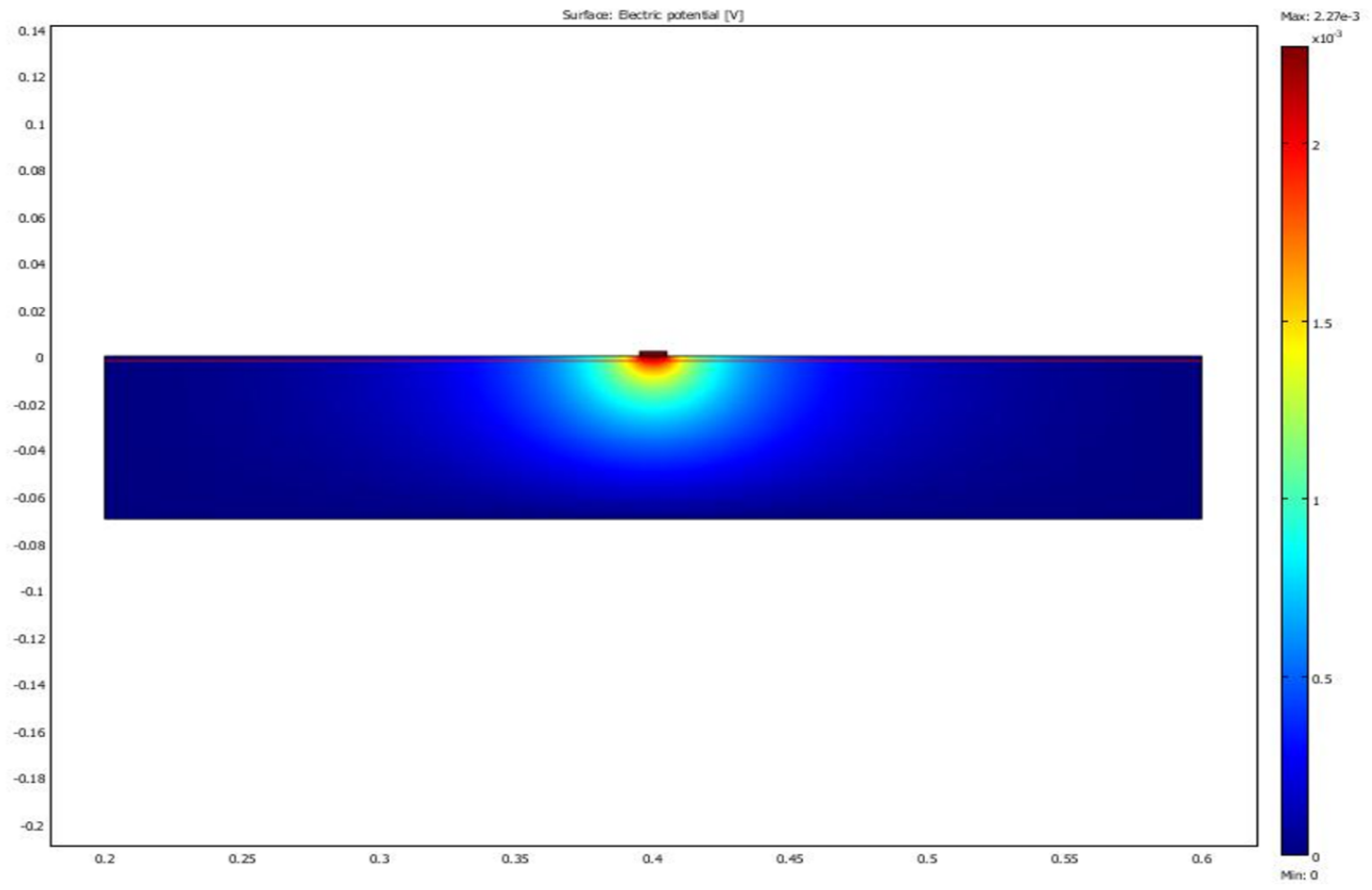

Figure 8: Homogenous isotropic model developed in COMSOL by Soto [17].

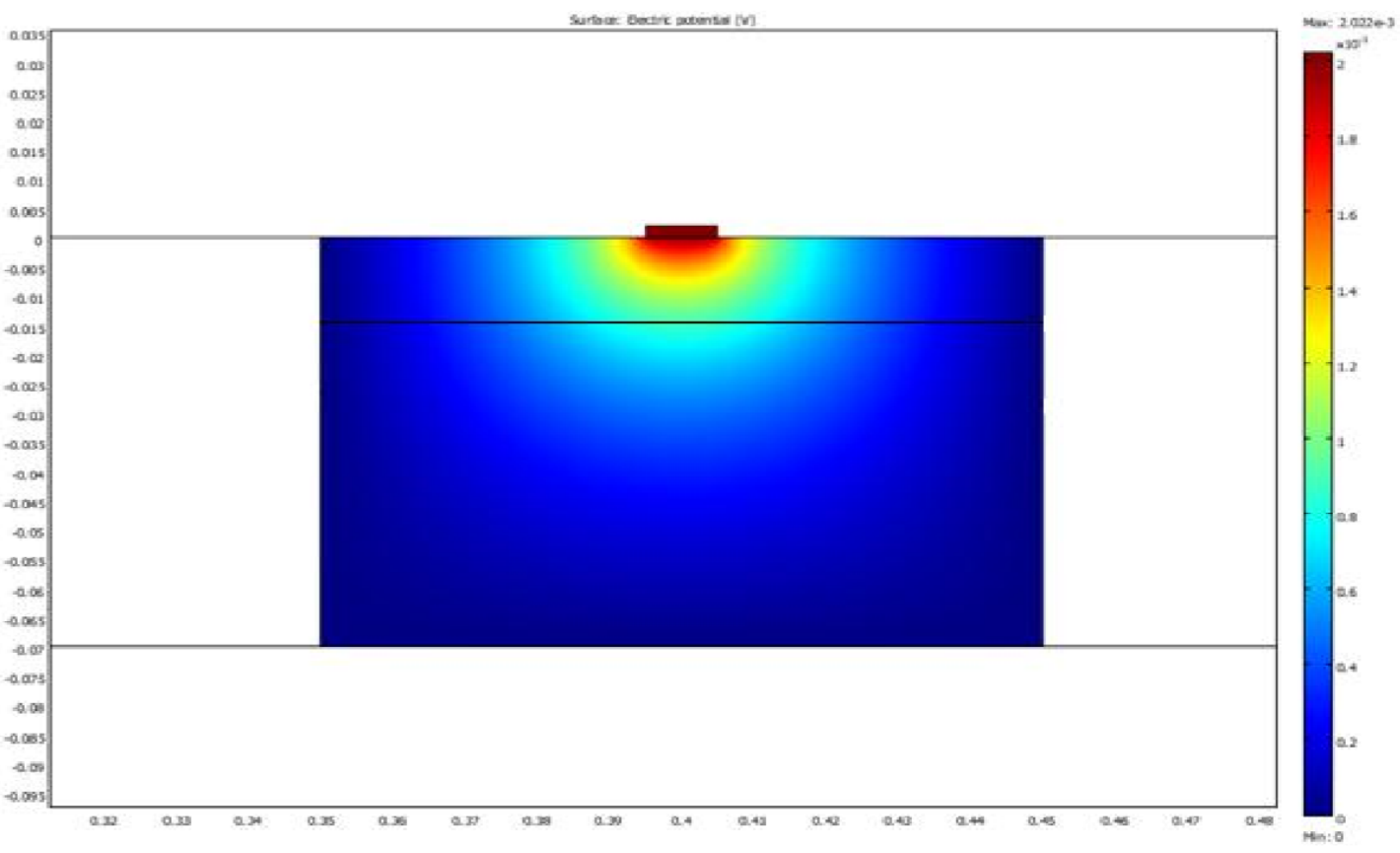

Figure 9: Non-homogenous isotropic model developed in COMSOL by Soto [17]. 
To vary fiber depth, Soto manually adjusted the position of the fiber geometry before performing analysis. It was found that changing the diameter of the fiber had a greater effect on the current stimulus than the fiber depth [17]. Soto's work concluded that "even the smallest change in the model can result in significant changes in current density required for nerve activation. This was shown by including the material properties of the fibers themselves to the model" [17].

Additional work currently being developed by Frank Sugden under the direction of Dr. Robert Szlavik is aimed at developing a model of neural membrane electrical characteristics coupled into a COMSOL model. This model is being developed using the Simulation Program with Integrated Circuit Emphasis (SPICE) platform. Spice is a general purpose simulation program for circuits, commonly used in electrical engineering [19]. To describe human nervous membrane potential characteristics, this model utilizes a Hodgkin-Huxley $(\mathrm{HH})$ circuit. The model incorporates both linear and non-linear circuit elements to describe the ion channel behavior of neurons. The myelinated neuron model created in SPICE is coupled with COMSOL to run a simulation. The COMSOL model consists of a two-dimension representation of the cross section of a human arm with stratified layers of skin, nerve, muscle, and bone. Figure 10 shows the basic geometry representation that this study utilizes. A strong approximate model was successfully developed, and validated with reference to the literature. Sugden's work will demonstrate that correct usage of neuron models within a two dimensional conductive space allows for approximate modeling of human neural electrical characteristics. Figure 11 shows an example of the human nerve membrane potential profiles that are used for validation. 


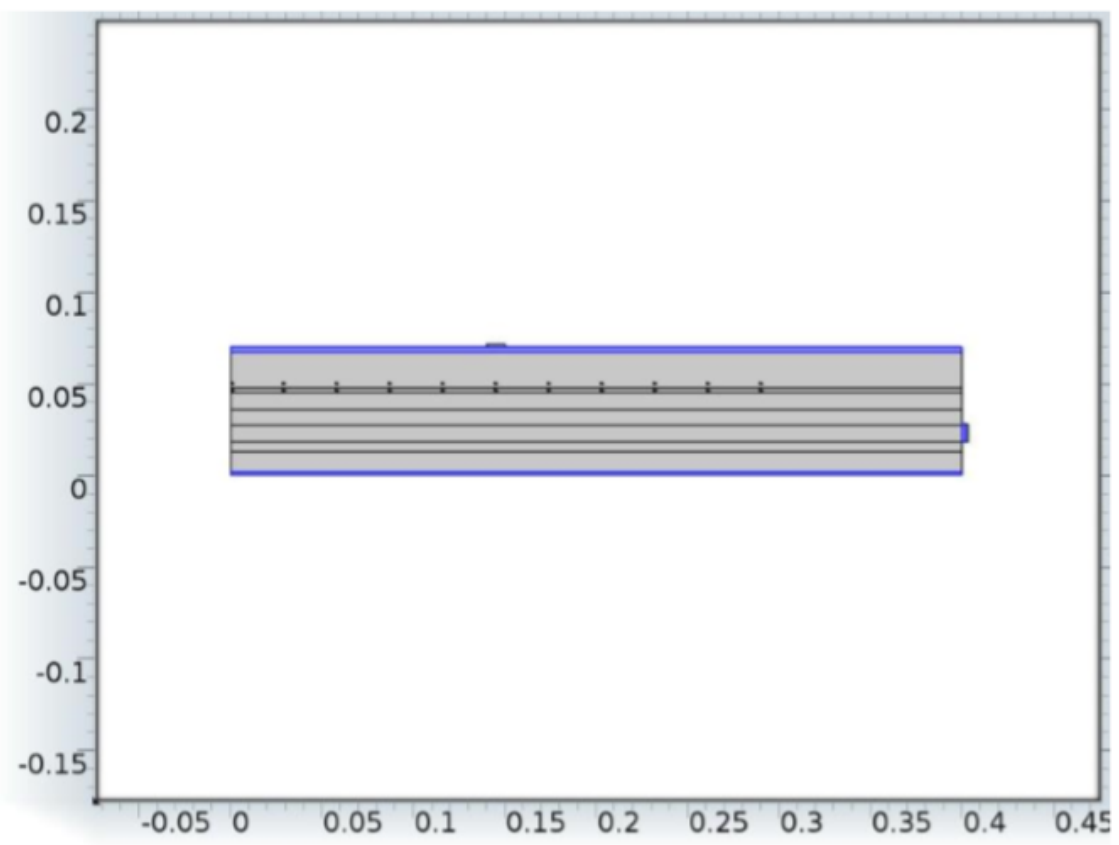

Figure 10: Two-dimensional geometry developed in COMSOL representing the human arm by Sugden.

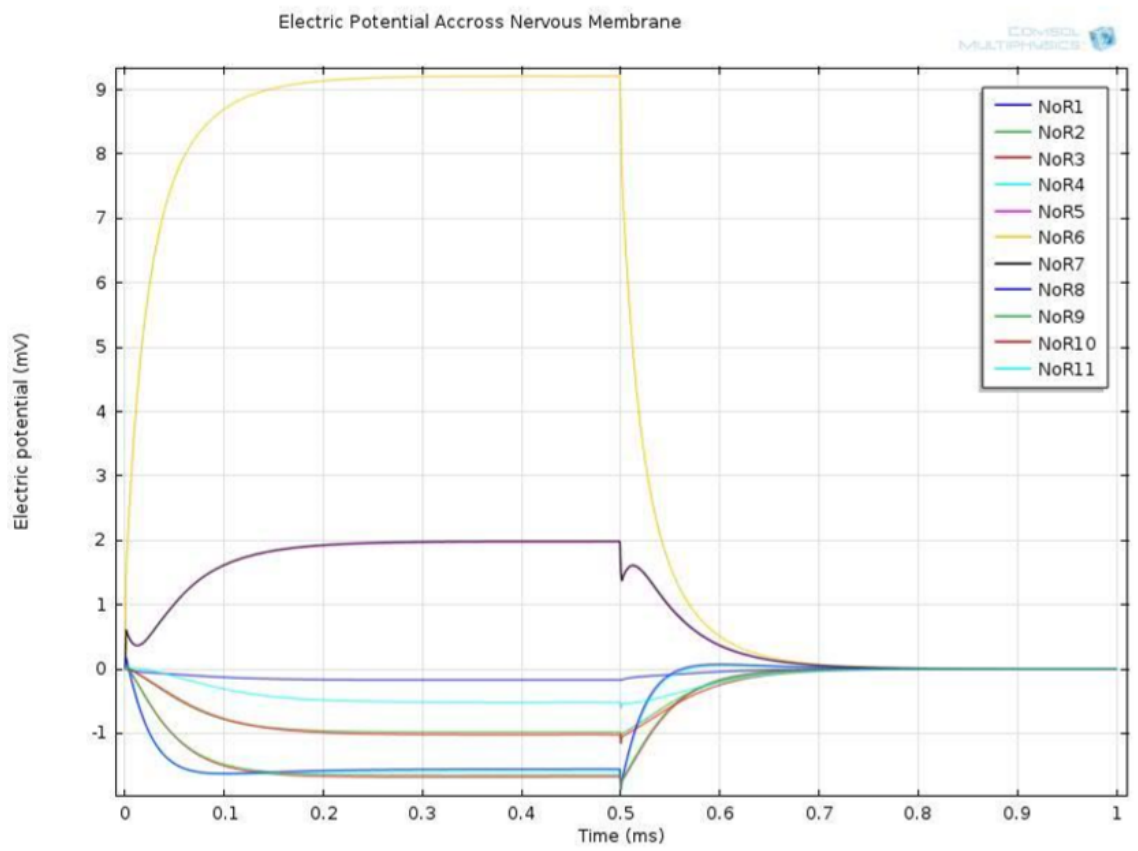

Figure 11: Human nerve membrane potential profile developed by Sugden.

These works illuminate the need for an anatomically correct three-dimensional model that can be incorporated into a simulation. Such a model can improve results and 
the understanding provided by nerve conduction studies. This formed the basis of the work defined in this thesis. 


\section{METHODS}

The goal of this work is to develop a geometrically accurate electric current volume conductor model for use with a SPICE model representing electrical behavior of nerve fiber membranes for characterizing nerve fiber activation. The complete model defined in this work is meant to support the SPICE netlist code. This work incorporates anatomical geometries and accurate tissue properties into a single model to serve as a test environment for current and future neural membrane models.

\subsection{Data Acquisition}

Data for the three-dimensional anatomically correct geometries was obtained from "BodyParts3D: 3D structure database for anatomical concepts" [20]. This openaccess project was funded by The Integrated Database Project and the Ministry of Education, Culture, Sports, Science and Technology of Japan. The goal of the project was to create "a dictionary-type database for anatomy in which anatomical concepts are represented by 3D structure data that specify corresponding segments of a 3D wholebody model for an adult human male" [20]. The database contains concepts for mapping materials with the purpose of computational manipulation, research, and experiments. Geometries for the database were constructed from a voxel human model for electromagnetic dosimetry, generated from a whole-body set of MRI images taken at $2 \mathrm{~mm}$ intervals [20]. The project culminated in an online database providing native 3D models of rendered body parts for download under a Creative Commons license. The online database is represented in a user-friendly graphical interface that allows body parts to be searched by location, depth, and size (Figure 12). The files are available for 
download in Wavefront OBJ format. The files opened in raw form are represented as a set of coordinates in a three-dimensional space. Figure 13 shows the right humerus bone in original Wavefront $\mathrm{OBJ}$ format.

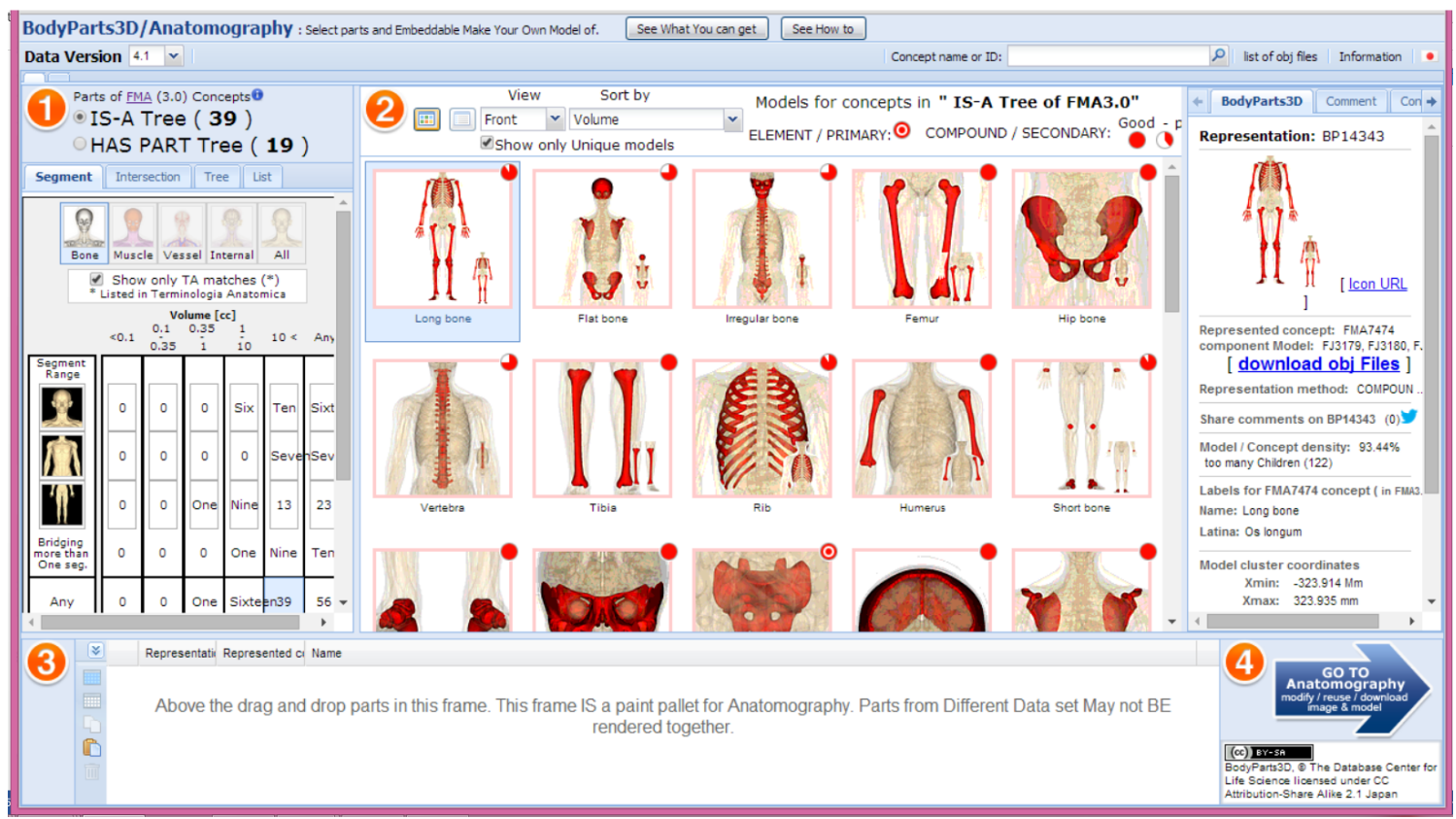

Figure 12: A screenshot of the BodyParts3D online database, which allows the user to select individual objects or organize by systems [20]. 


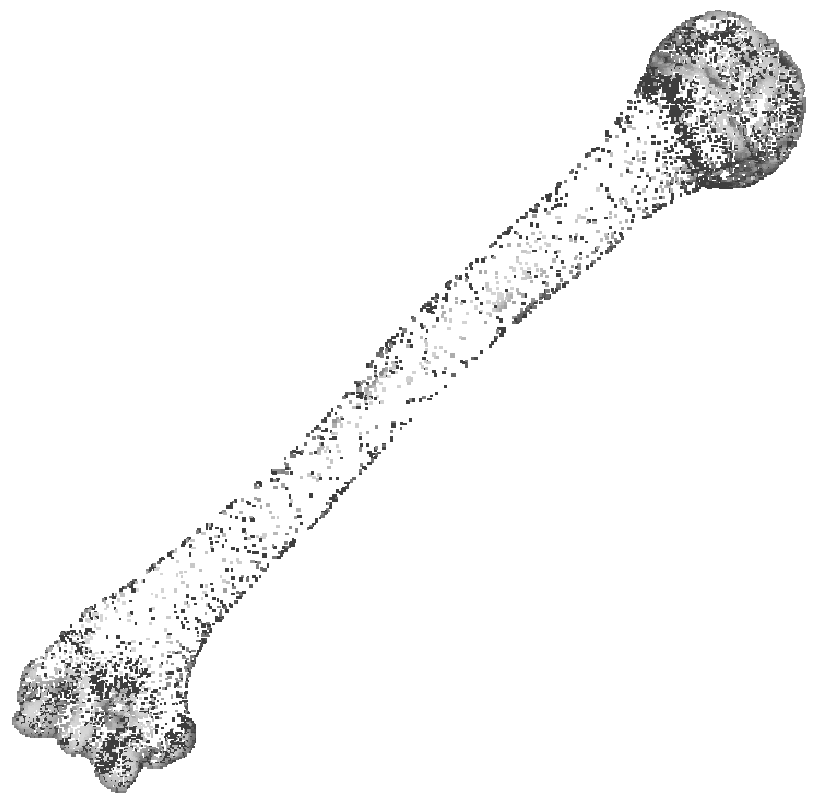

Figure 13: Raw file of the right humerus bone obtained from BodyParts3D in Wavefront OBJ format.

The original files obtained from BodyParts3D were opened using a program titled Meshlab (available from meshlab.org) [21]. Meshlab is "an open source, portable, and extensible system for the processing and editing of unstructured 3D triangular meshes" [21]. This program was designed to aid the processing of large, unstructured models obtained from 3D scanning and provides tools for editing, inspecting, and converting meshes [21].

Once files obtained from BodyParts3D were opened in Meshlab, a mesh of 3D triangles was generated. The initial generated mesh of the right humerus bone can be seen in Figure 14. The initial meshes that were imported from raw data provided by BodyParts3D contained errors such as holes, border edges, and invalid orientations. To repair the mesh, fill holes, and clean irregular triangles, the mesh generated in Meshlab was exported in the Stereo Lithography (STL) file format. 


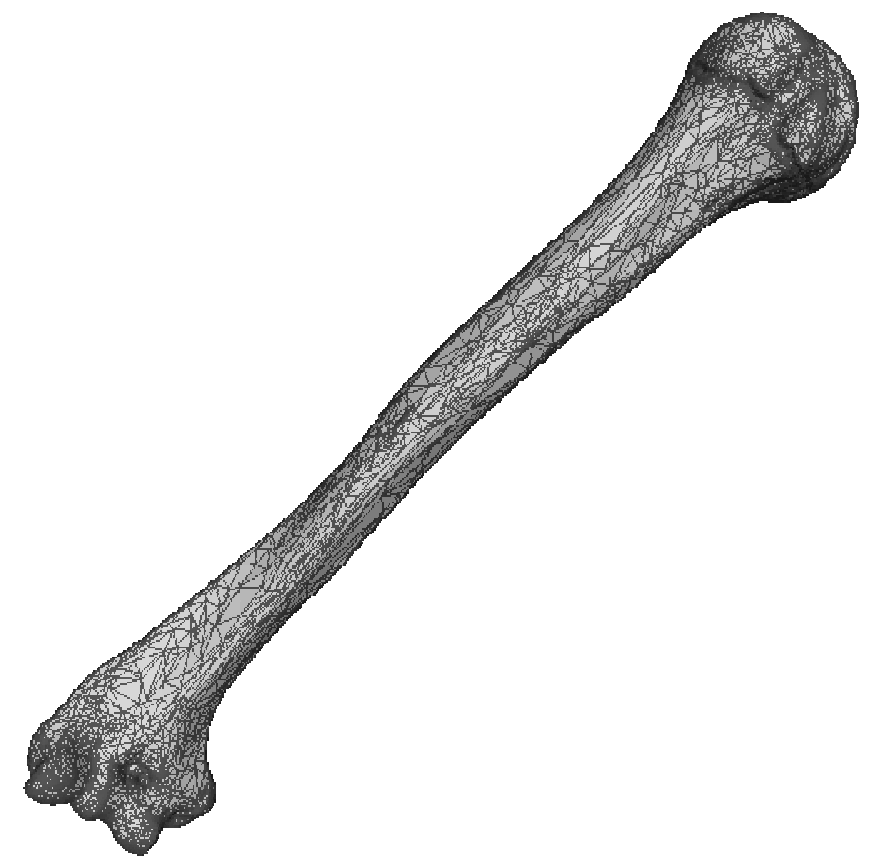

Figure 14: Initial mesh generated on the right humerus bone in Meshlab.

Each STL file was then opened in a program called netfabb Basic. This program is a free program available for mesh edit, repair, and analysis originally created for Additive Manufacturing (also known as 3D Printing). Once in netfabb Basic, each mesh was automatically repaired. Figure 15 shows the right humerus bone in netfabb Basic during editing. Next, the mesh was visibly inspected for spikes, holes, and areas of excessive complexity and corrected by hand. Each file was saved as a final STL and was then ready for import into COMSOL. 


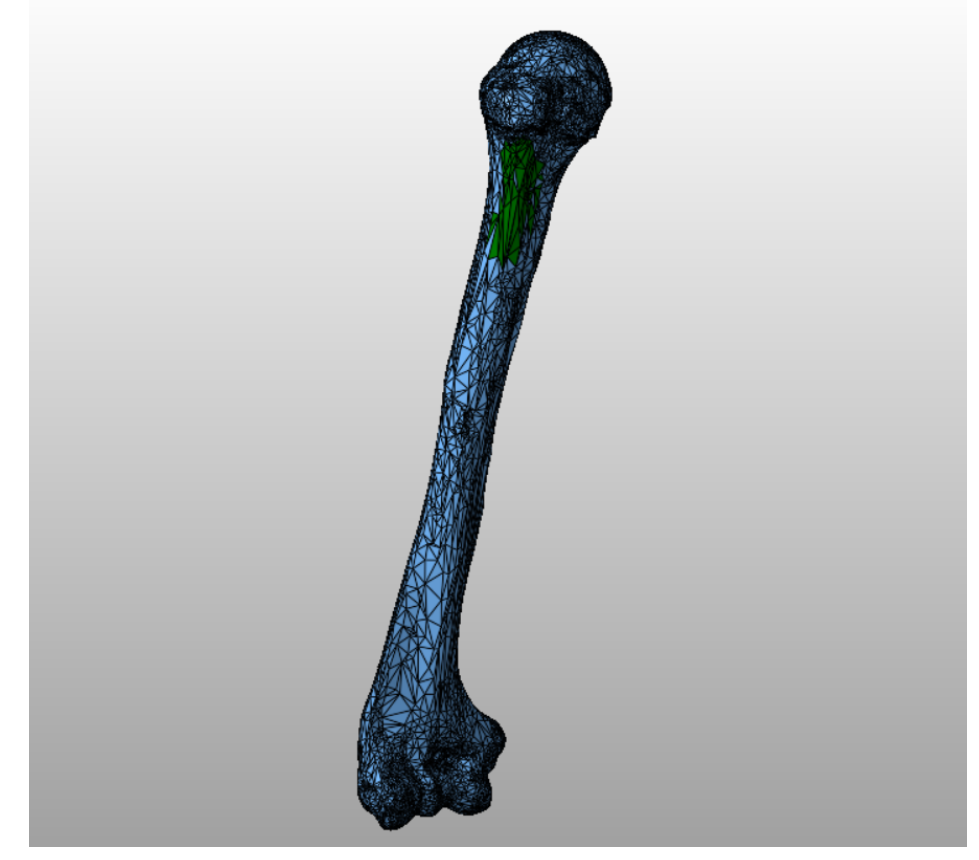

Figure 15: The right humerus bone in netfabb Basic during mesh editing.

\subsection{Model Development}

This model was developed following the computational modelling process previously outlined in Figure 5 and defined by the FDA Guidance Document [13]. A summary of the steps specific to this model are outlined in Figure 16. 


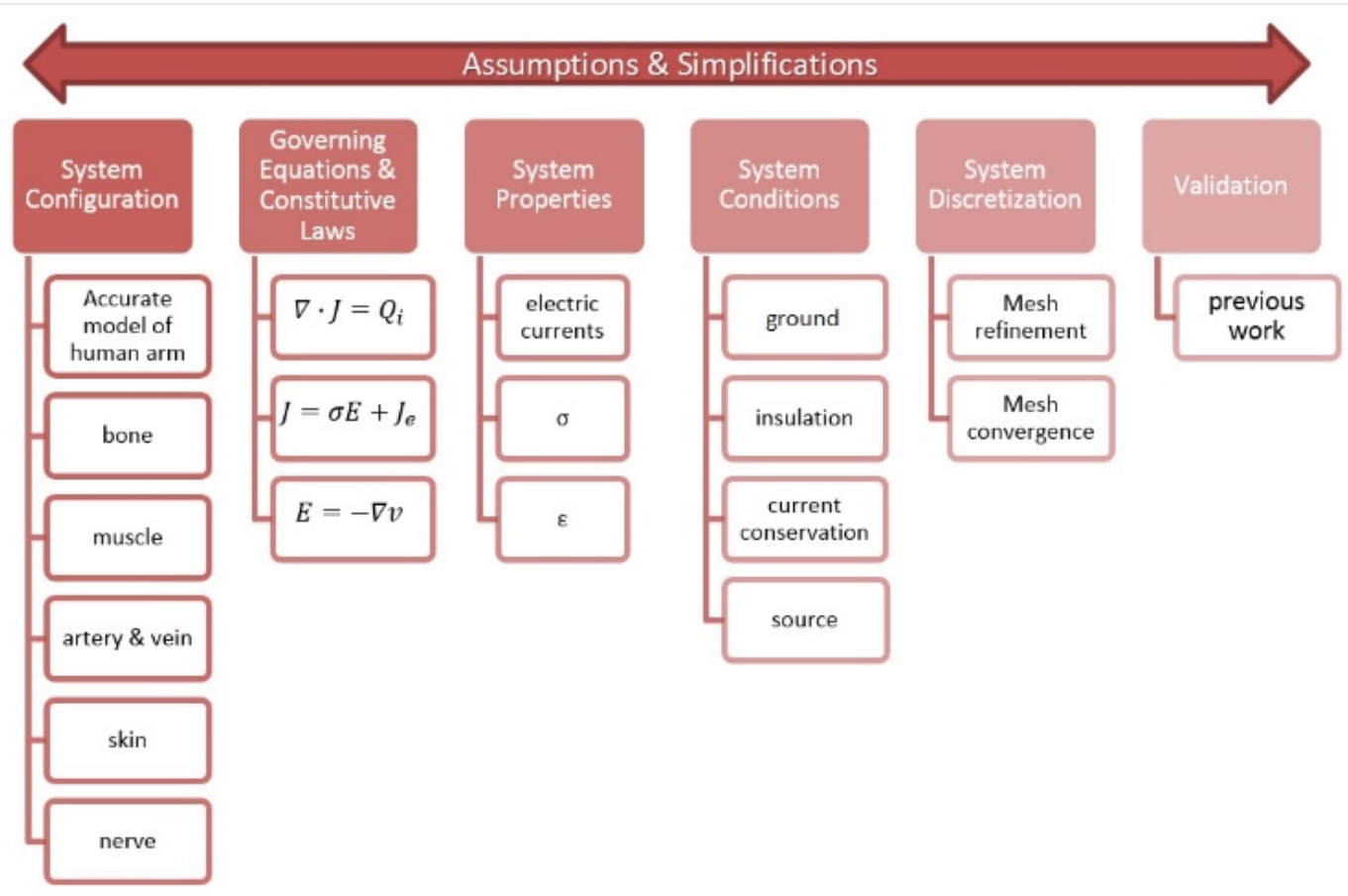

Figure 16: The six steps to developing a computational model, as in this work.

\subsubsection{System Configuration}

The goal of this system is to create an accurate model of the human arm. The scope of this model is limited to the right arm, cut mid-bicep. All major materials found in the arm are represented including bone, muscle, skin, artery and vein. Nerve is represented as a system of small anatomically positioned spheres. Materials for fat and cartilage were excluded from this model in order to optimize model tractability. The bones represented in this model include the following: humerus, radius, ulna, scaphoid carpal, lunate carpal, triquetrum carpal, pisiform carpal, trapezoid carpal, capitate carpal, hamate carpal, the five metacarpals, distal phalanges, middle phalanges, and proximal phalanges. A complete assembly of the bones used for import into COMSOL in the model can be found in Figure 17. 


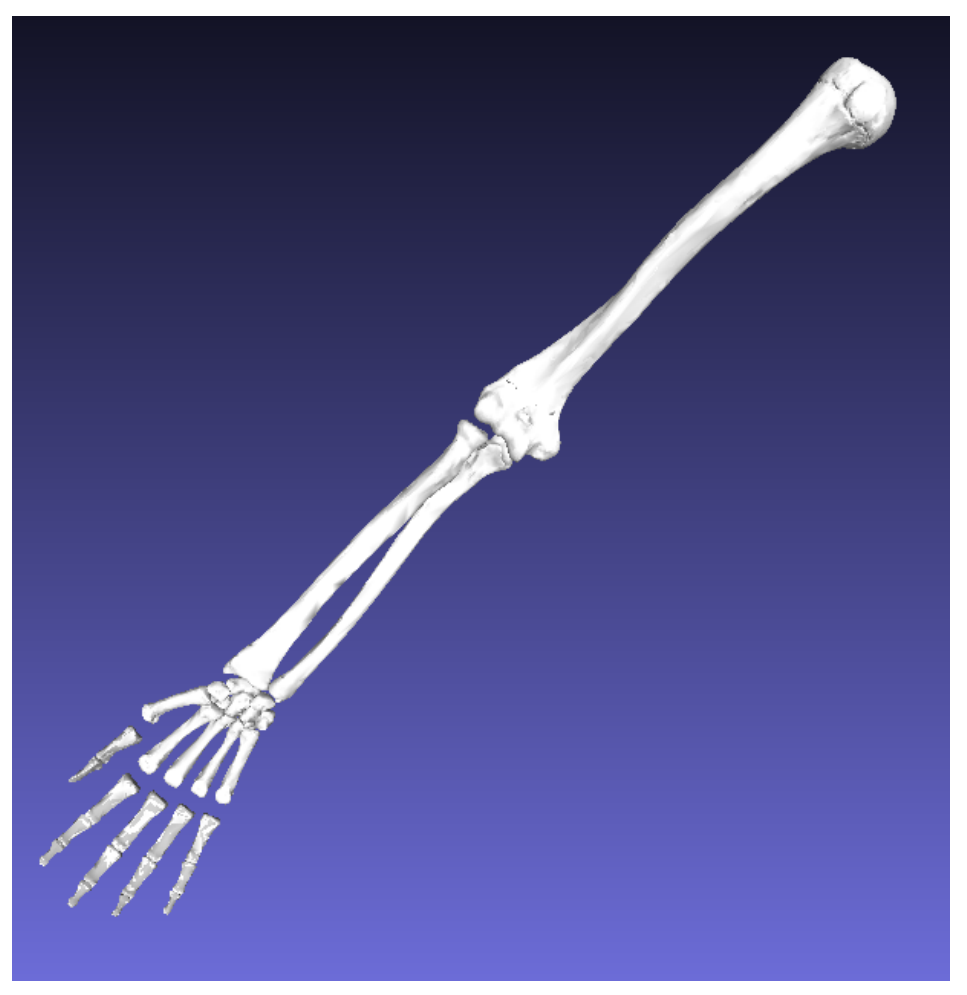

Figure 17: Complete assembly of the bones of the human arm, ready for import into COMSOL.

The muscles represented in this model include the following: triceps brachii, biceps brachii, brachialis, brachioradialis, anconeus, pronator teres, flexor carpi radialis, palmaris longus, flexor carpi ulnaris, flexor pollicis longus, pronator quadratus, supinator, flexor digitorum superficialis, flexor digitorum profundus, lumbricals, thenar muscles of thumb, extensor carpi radialis longus, extensor carpi radialis brevis, extensor digitorum, extensor carpi ulnaris, abductor pollicis longus, extensor pollicis brevis, extensor pollicis longus, extensor indicis, abductor pollicis longus, extensor pollicis longus, extensor pollicis brevis, extensor indicis, opponens pollicis, and opponens digiti minimi. A complete assembly of the muscles used for import into COMSOL in the model are shown in Figure 18. 


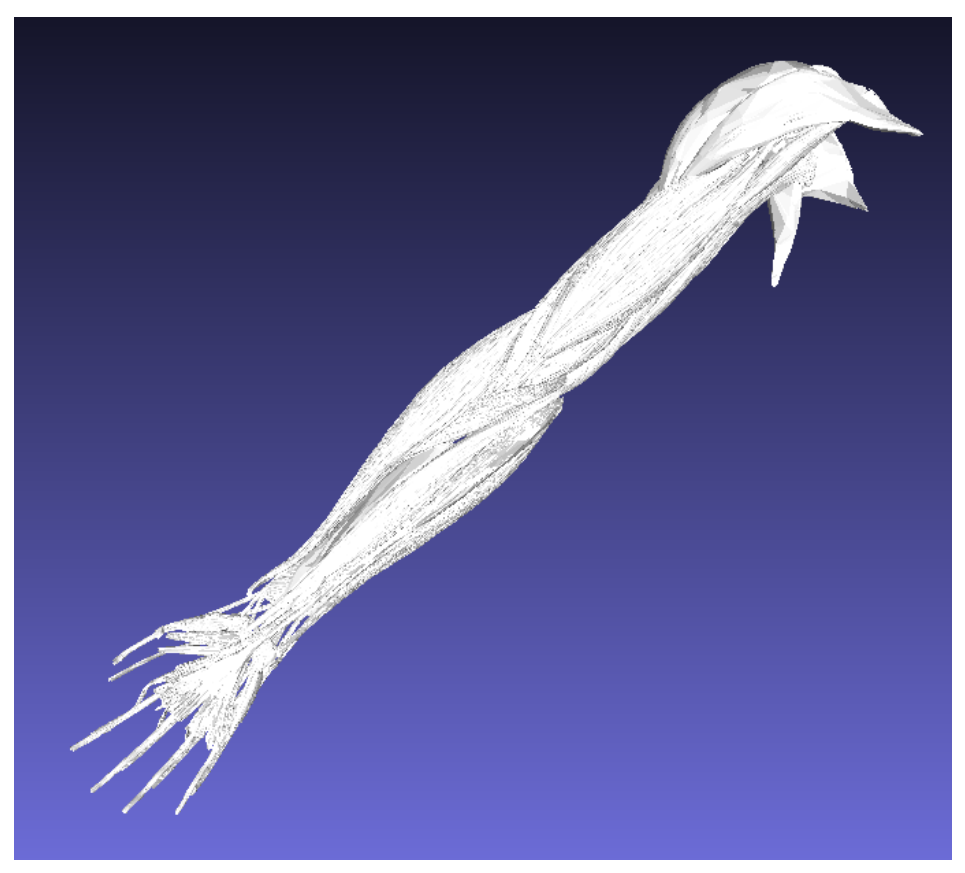

Figure 18: Complete assembly of the muscles of the human arm ready for import into COMSOL.

Arteries and veins represented in this model include the following: axillary artery, brachial artery, radial artery, ulnar artery, deep palmar arch, superficial palmar arch, digital arteries, cephalic vein, brachial vein, basillic vein, median cubital vein, ulnar vein, radial vein, digital veins. A complete assembly of the arteries and veins used for import into COMSOL in the model can be seen in Figure 19. Data for skin from BodyParts3D was represented by a single file. The skin representation was sliced using netfabb Basic at mid bicep level. The final skin geometry used for this model can be seen in Figure 20. 


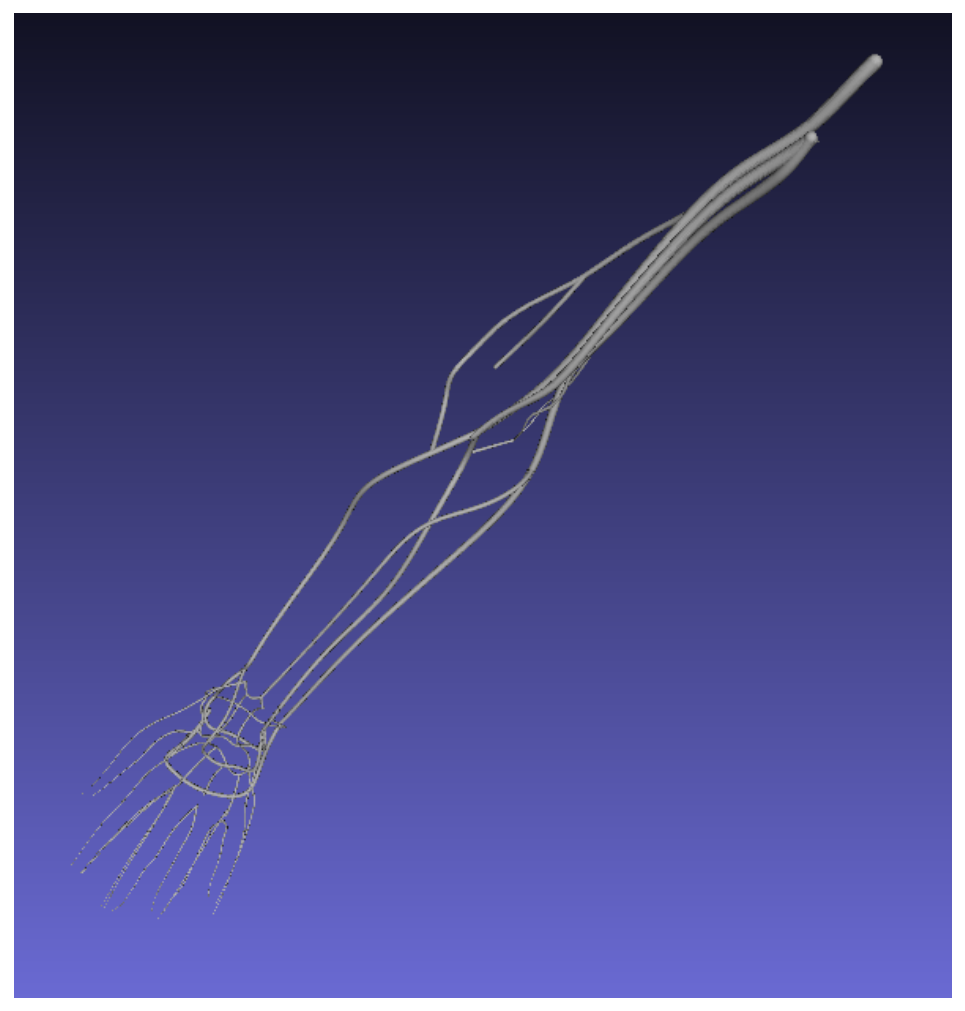

Figure 19: Complete assembly of the artery and vein of the human arm ready for import into COMSOL.

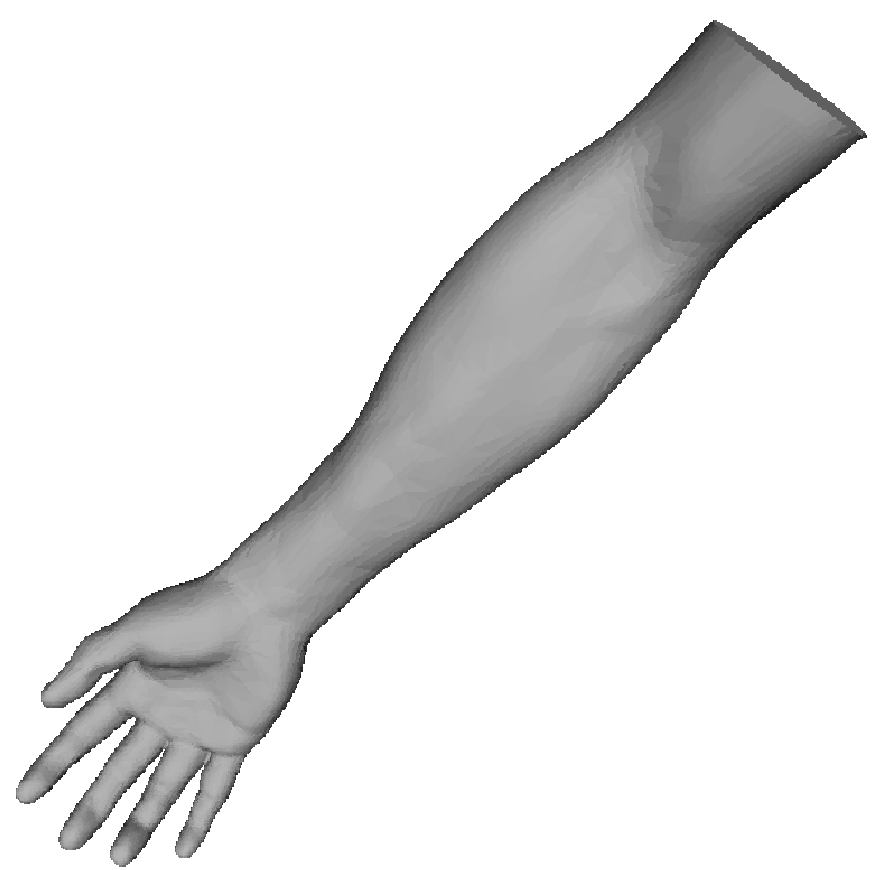

Figure 20: Final skin geometry, ready for import into COMSOL. 
Each of the STL files containing the assembly for each tissue type was uploaded into COMSOL using the import module. The geometries are highly complex, containing many faces, making importing a difficult process. The CAD Import Module kernel with a relative repair tolerance of 1e-10 was selected because of its ability to read files created in other programs. For individual file import, only surfaces were selected as objects to import. The "knit surfaces" option was selected so that solid surfaces could be formed. An absolute import tolerance of 1e-10 was chosen for each import. The final geometry composition containing bones, muscles, skin, arteries and veins can be seen in Figure 21. The final geometry composition with skin hidden from view to display the other anatomical structures can be seen in Figure 22.

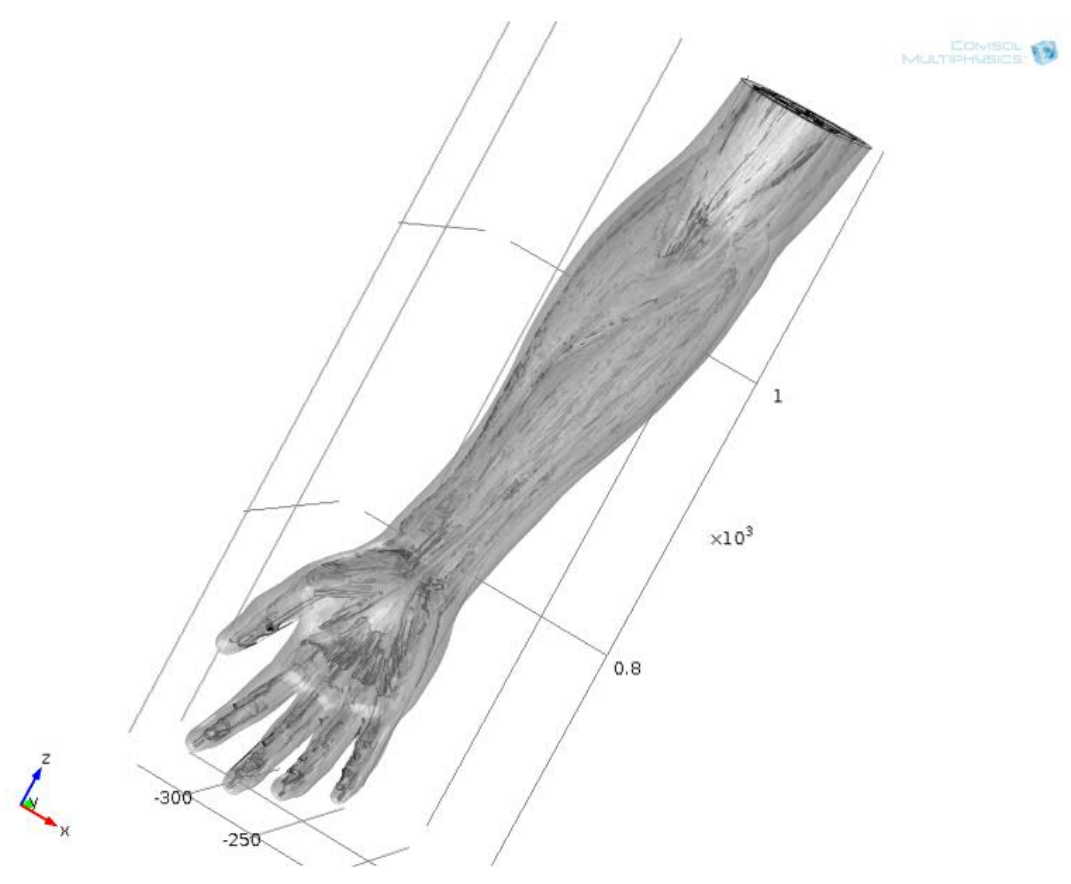

Figure 21: Final geometry composition including bone, muscle, skin, artery, and vein with transparency enabled to enhance viewing. 


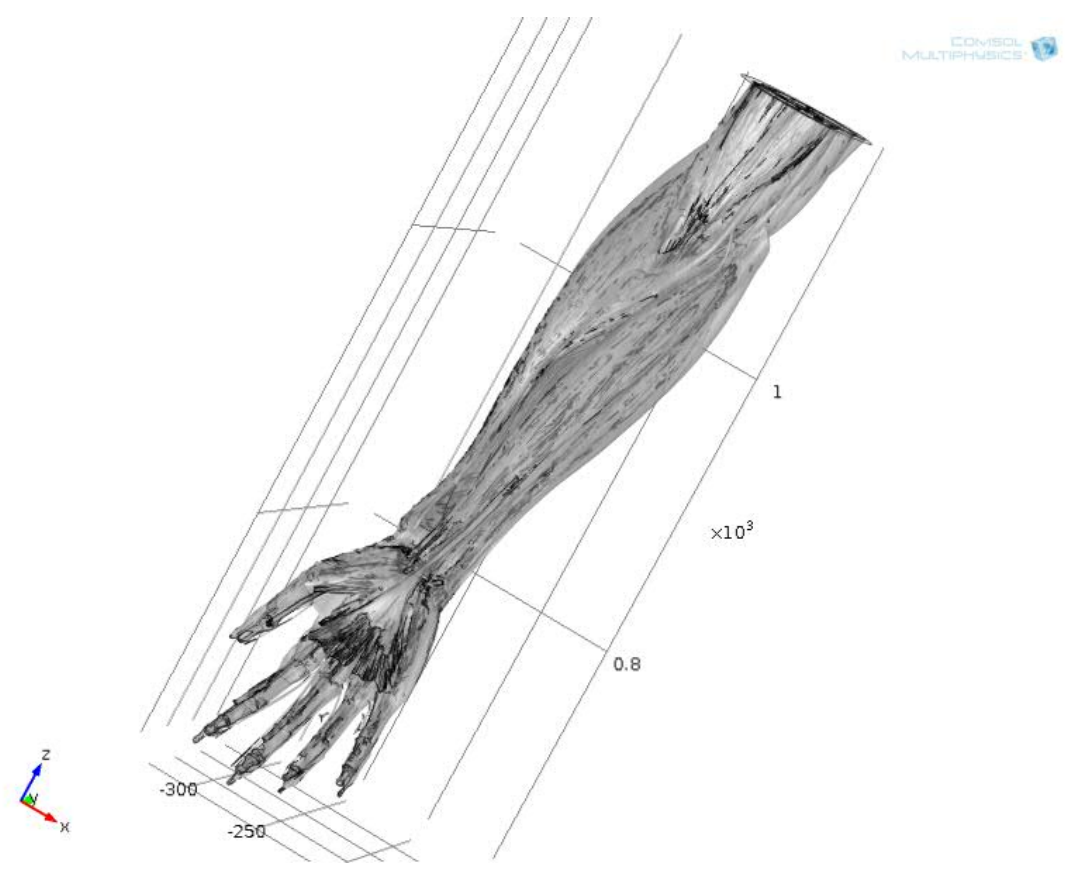

Figure 22: Final geometry composition including bone, muscle, artery, and vein with skin hidden from view to enhance viewing.

Finally, twenty-two small spheres were drawn into the model in COMSOL to represent the presence of nerve. Nerve was represented by two chains of eleven spheres. These spheres utilized the assumption that nerve tissue in this model is homogenous in the lateral position. This means that each sphere couple occupies the same $\mathrm{Y}$ and $\mathrm{Z}$ coordinates, and was only varied in the X. Within a sphere couple, one was placed close to the membrane where nerve would be present, and the second was placed close to the first but within the muscle subdomain. The nerve tissue was drawn in this way for two reasons. First, the absence of nerve in BodyParts3D files created a need for the presence of nerve fiber geometries where material properties could be applied. Second, this specific structure of nerve represented by eleven sphere couples allows for easy integration with the SPICE netlist code developed to model neural membrane electrical characteristics. 


\subsubsection{Governing Equations \& Constitutive Laws}

This model utilizes the Electric Currents physics module available in COMSOL. The Electric Currents module adds corresponding equations, boundary conditions, and current sources for modeling electric currents in conductive media [14]. Each boundary condition and node applied to this module adds or adjusts the governing equations affecting the simulation. The Electric Currents module in COMSOL utilizes two basic governing equations:

$$
\nabla \cdot J=Q_{i}
$$

where $J$ is current density in amperes per meter squared, and $Q_{i}$ is the initial current source in amperes per meters cubed.

$$
J=\sigma E+J_{e}
$$

where $\sigma$ is electrical conductivity in siemens per meter, $E$ is the electric field in volts per meter $J_{e}$ is externally generated current density in amperes per meter squared. These basic equations are applied to the entire model, except where boundary conditions or nodes are applied.

Adding a current conservation node to the model adds the continuity equation for the electrical potential [14]. Electrical conductivity and relative permittivity are used in this node for the displacement current. The equations applied to this node are:

$$
\begin{gathered}
\nabla \cdot J=Q_{i} \\
J=\sigma E+J_{e} \\
E=-\nabla V
\end{gathered}
$$

where $V$ is the electric potential in volts. 
Adding the electrical insulation boundary condition means that no electric current flows into the boundary. The equation applied at this boundary is:

$$
n \cdot J=0
$$

Adding a boundary current source boundary condition adds a current source on interior boundaries which can represent a source or sink of current. The equation applied at this boundary is:

$$
n \cdot\left(J_{1}-J_{2}\right)=Q_{j}
$$

where $Q_{j}$ is the current source in amperes per meters cubed.

The ground node boundary condition applies a boundary as ground. At ground, there is a zero potential condition. The equation applied at this boundary is:

$$
V=0
$$

These equations are applied at thousands of points throughout the model. This results in a large system of equations, which COMSOL solves after forming matrices.

\subsubsection{System Properties}

To create an accurate volume conductor model, realistic tissue properties must be used. However, material property characterization of biological tissues can be difficult. Many studies have been conducted both in-vitro and in-vivo in an attempt to correlate functions or values to describe material properties. In this study, each geometric subdomain representing a single tissue was assigned a relative permittivity and electrical conductivity to apply dielectric properties. 
Several assumptions were made when determining what values to use for characterizing the dielectric properties of materials for this model. First, the notion of dependent functions to represent a dielectric property was disregarded, and independent values were used instead. Dependent functions used to describe material properties are not scalar values, but instead vary with other factors in the model such as temperature, frequency, or time. How to accurately characterize the dielectric properties of biologic tissues is still widely unknown and is the focus of many studies [22, 23]. For this model, dependent functions would greatly add to computation time and simulation complexity. Arteries and veins were assumed to be homogenously comprised of blood, and the properties of vascular walls were disregarded. Skin was assumed to be a homogenous material, disregarding the stratum layers of the skin. Muscles was assumed to be entirely longitudinal and transverse components were disregarded. Bone was assumed to be a homogeneous material, and the dielectric properties for cortical bone were used. A summary of the properties used in this study can be found in Table 1. For materials that display a highly frequency-dependent nature, the frequency of $433 \mathrm{MHz}$ was assumed. This is a standardized frequency used in Industrial Scientific Medical applications. 


\begin{tabular}{|c|c|c|c|}
\hline Tissue & $\begin{array}{c}\text { Relative Permittivity } \\
\varepsilon\end{array}$ & $\begin{array}{c}\text { Electrical Conductivity } \\
\qquad \sigma[\mathrm{S} / \mathrm{m}]\end{array}$ & Reference \\
\hline Muscle & 64.21 & 0.333 & {$[24,25]$} \\
\hline Artery \& Vein & 57.3 & 0.667 & {$[24,25]$} \\
\hline Skin & 42.48 & 0.5 & {$[24,25]$} \\
\hline Bone & 13.77 & 0.1032 & [24] \\
\hline Nerve & 35.7 & 0.500 & [24] \\
\hline
\end{tabular}

\section{Table 1: Summary of the dielectric properties applied for each tissue in COMSOL including sources for each property.}

A new material node was created for each tissue incorporated into the model. A total of five material nodes were included; skin, muscle, bone, nerve, and vein. The corresponding relative permittivity and electrical conductivity for each tissue was applied to each material node. Applying material properties using this method ensures that the correct dielectric properties are applied to each tissue consistently throughout the model.

\subsubsection{System Conditions}

Nodes and boundary conditions form the basis of how equations and conditions are applied to each model. Biological principals and behavior of the system dictate how and where boundary conditions and module nodes are applied.

Current conservation was applied to each separate tissue subdomain. A total of five current conservation nodes were used. Individual current conservation nodes for each tissue utilized the corresponding tissue properties of that subdomain. Electric insulation was applied to every external boundary excluding the cross-section of the arm where the bicep was sectioned (Figure 23). This boundary condition mimics the presence of 
external skin. Due to the complexity of geometries, over a thousand individual boundaries received the electric insulation condition. Ground was applied to the crosssection of the arm where the bicep was sectioned (Figure 24) [20, 26]. This is the only external boundary on the model that represents an internal cross-section of the arm. This boundary includes sections of skin, muscle, bone, and vein. The Boundary Current Source condition was applied to the internal boundary on the electrode that intersects skin (Figure 25). The skin-electrode interface is where the source is realistically introduced into the system. Only one boundary received this condition.

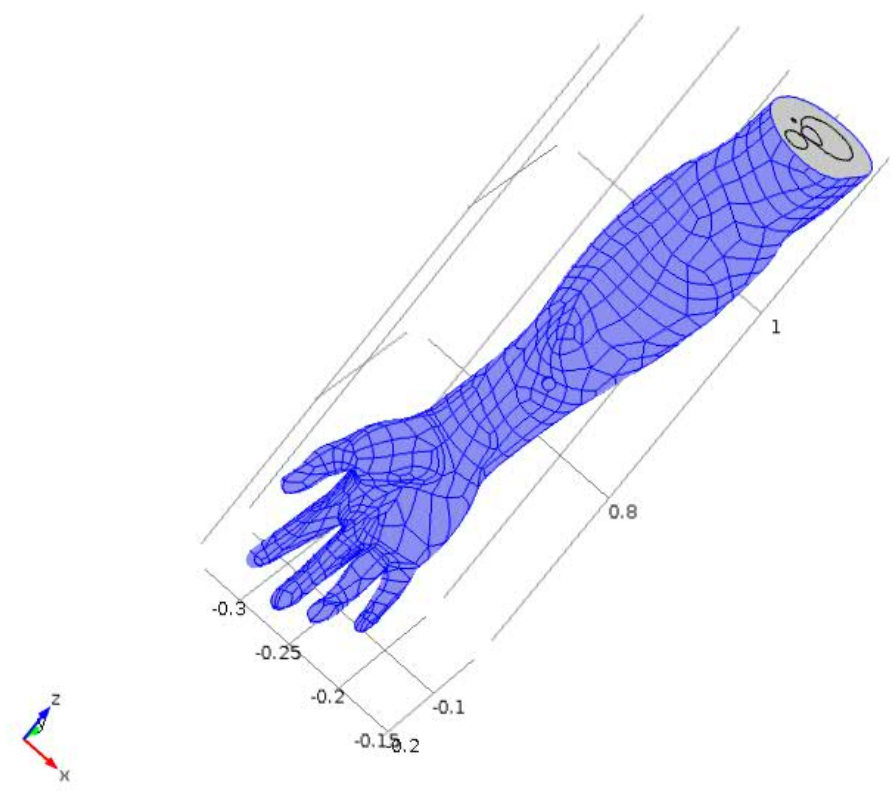

Figure 23: Electric insulation boundary condition applied at all external surfaces of the arm, represented in purple. 


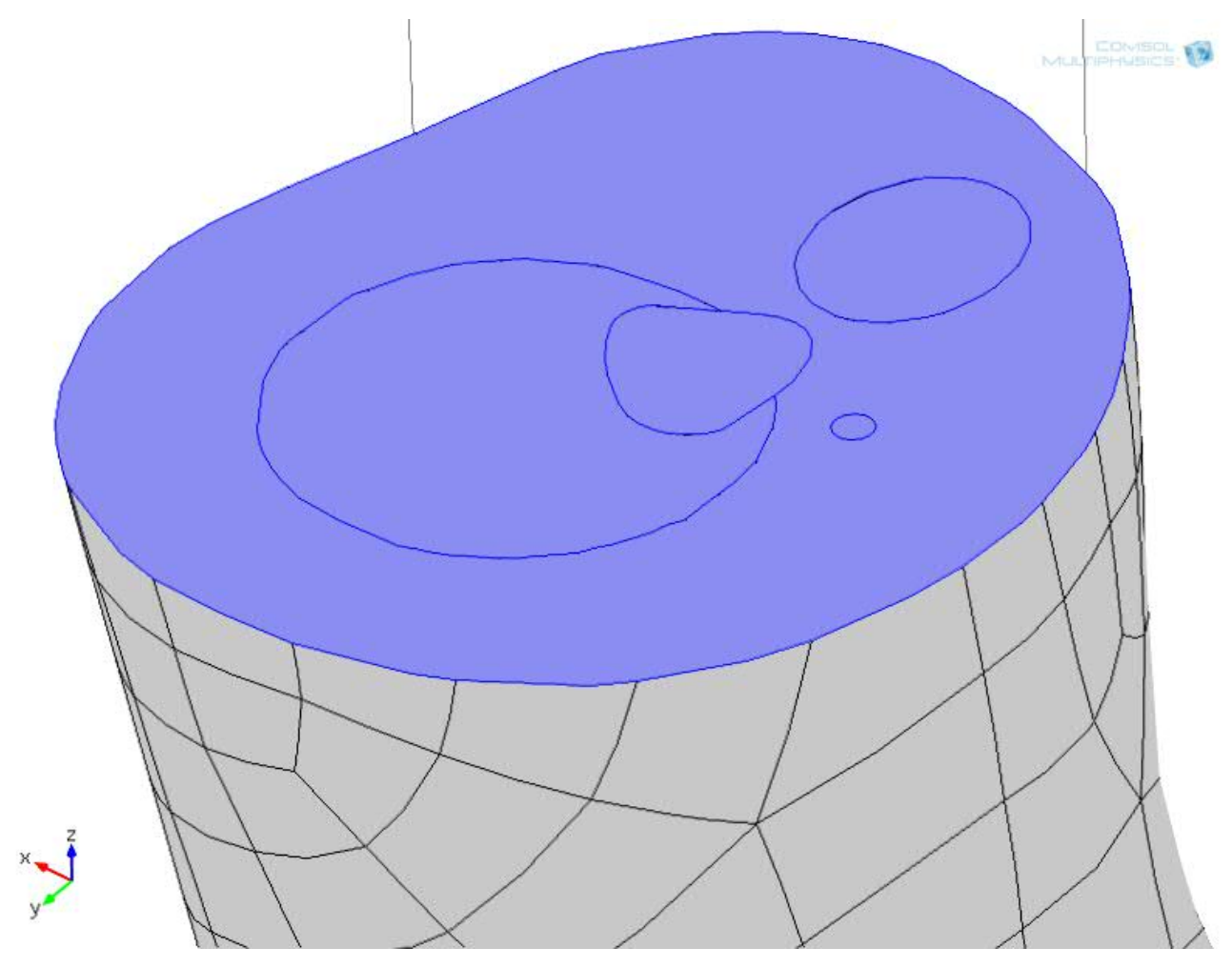

Figure 24: Ground boundary condition applied at the cross-section of the human arm (mid-bicep), represented in purple. 


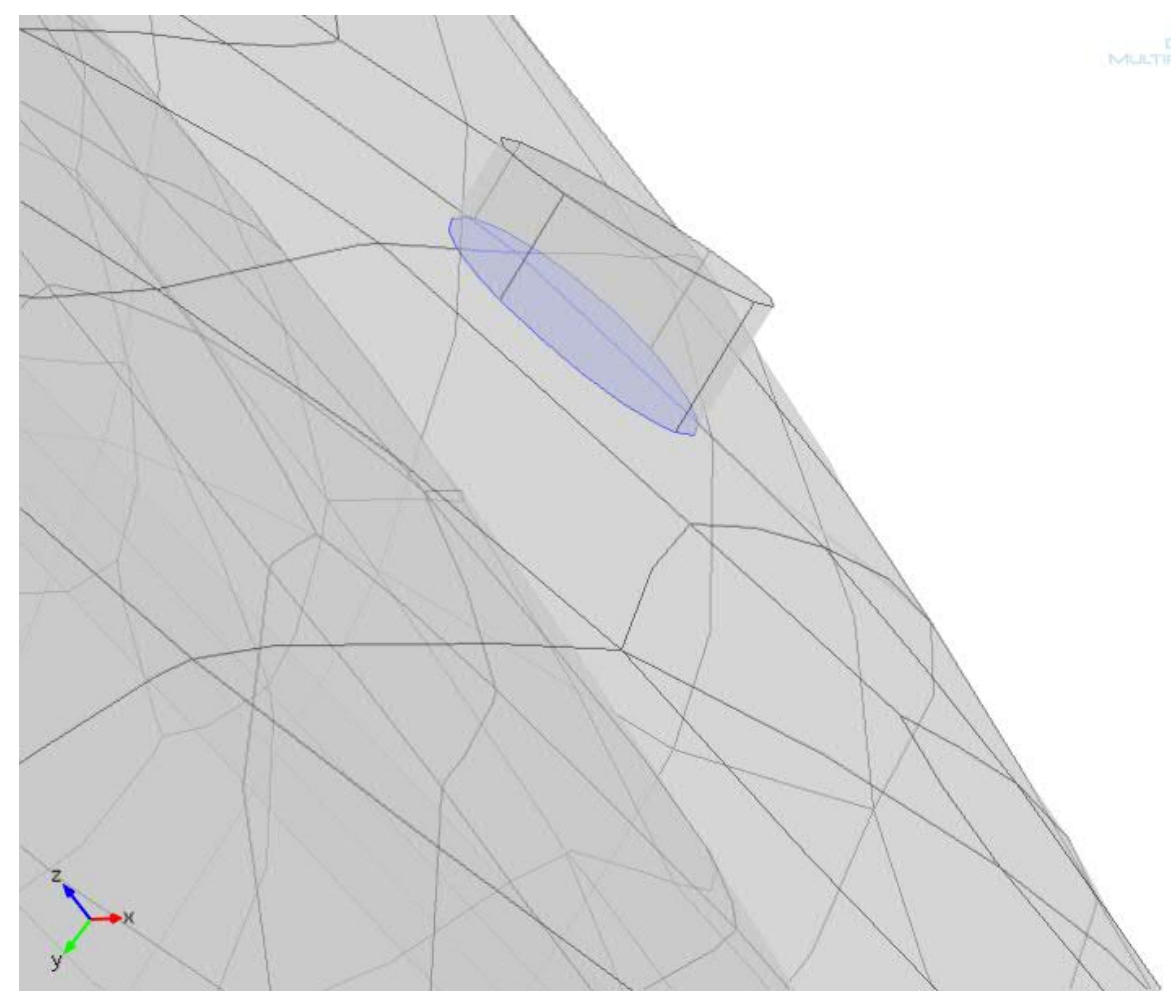

Figure 25: Current source boundary condition applied at the contact between skin and electrode, represented in purple.

\subsubsection{System Discretization}

COMSOL provides many different solvers and study options to customize how your system will be discretized. Study steps including stationary, time dependent, parametric sweep, and many more can be added to a study and customize how the simulation is solved. The user also has the ability to select the solver used to compute results. These solvers include but are not limited to: stationary, time-dependent, parametric sweep, and time discrete.

Due to computational limitations discussed later in Section 2.3, a stationary study was preformed instead of the originally intended time-dependent study. The Stationary Solver was selected with a relative tolerance of 0.001 . A Direct node with the 
Multifrontal Massively Parallel sparse direct Solver (MUMPS) and a memory allocation factor of 1.2 was used to handle settings for the direct linear system solver.

\subsubsection{Mesh Development}

A critical component of an optimized computational model lies in the mesh development. This model was particularly challenging to mesh due to the abnormal geometries representing biologic materials. Sizes ranged from small blood vessels to the large skin component, and many geometries intersected, touched, or presented miniscule spaces between them. Originally, the entire model was meshed in Meshlab, where individual elements could be easily controlled. Meshlab has the capability to mesh many complex geometries fairly quickly and has more computational power than COMSOL's built-in mesh tool. Meshes for the entire model were successfully imported into COMSOL (Figure 26). However, it was discovered that once a mesh is imported into COMSOL, there is no ability to add boundary conditions and physics to the geometry. The final mesh for this model was developed using the COMSOL mesh tool. 


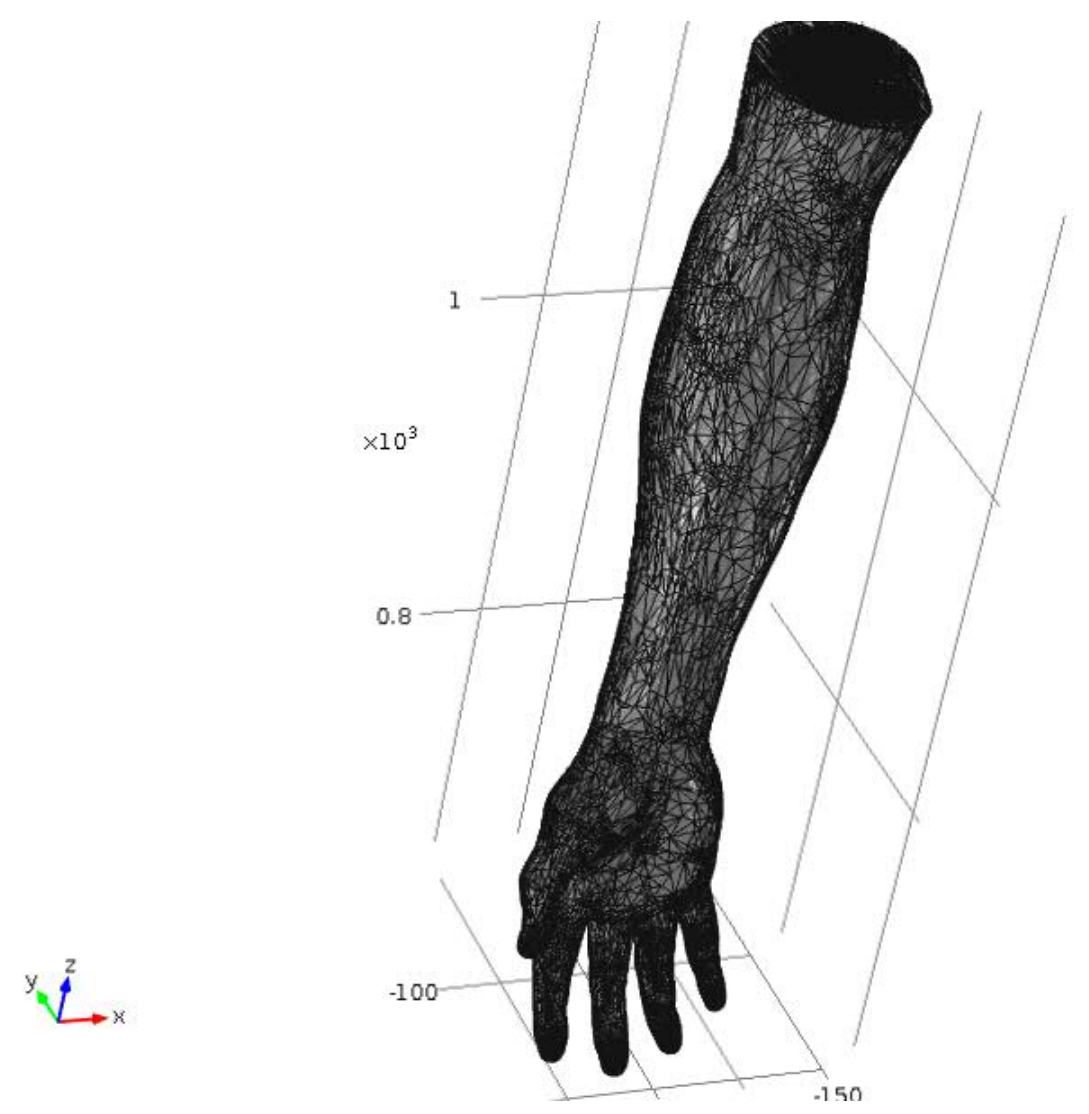

Figure 26: Complete assembly of the human arm, pre-meshed in Meshlab and imported into COMSOL.

Mesh elements must be small enough to provide an accurate result, yet not so fine that computational power is wasted. To determine at what element size the simulation would run most optimally, a mesh convergence study was performed. To develop a mesh convergence, mesh element numbers were varied, and corresponding electric potential of a single point was recorded. Using COMSOL's basic mesh tool, mesh element size can be set to a predefined size by selecting a setting ranging from "extremely fine" to "extremely coarse." These basic settings in combination with manual mesh refinements were used to generate the appropriate range of element numbers to determine convergence.

\subsection{Model Simplification}


The complexity of the geometries of this model posed a great difficulty in developing a mesh and computing the solution. As discussed earlier, challenges were encountered in the geometry import, system discretization, and mesh development. The biological systems of the bone, blood vessel, skin, and particularly muscle are inherently complex in nature. Muscle is not a simple object, but is instead composed of many fibers (Figure 27). The organization of muscle follows a complex fibrous organization. The fiber-like nature of muscle was visible in the geometric entities obtained from BodyParts3D. This challenge was initially overcome by manually repairing the STL files. However, once imported into COMSOL these detailed and complex files became a burden to the functionality of the software.

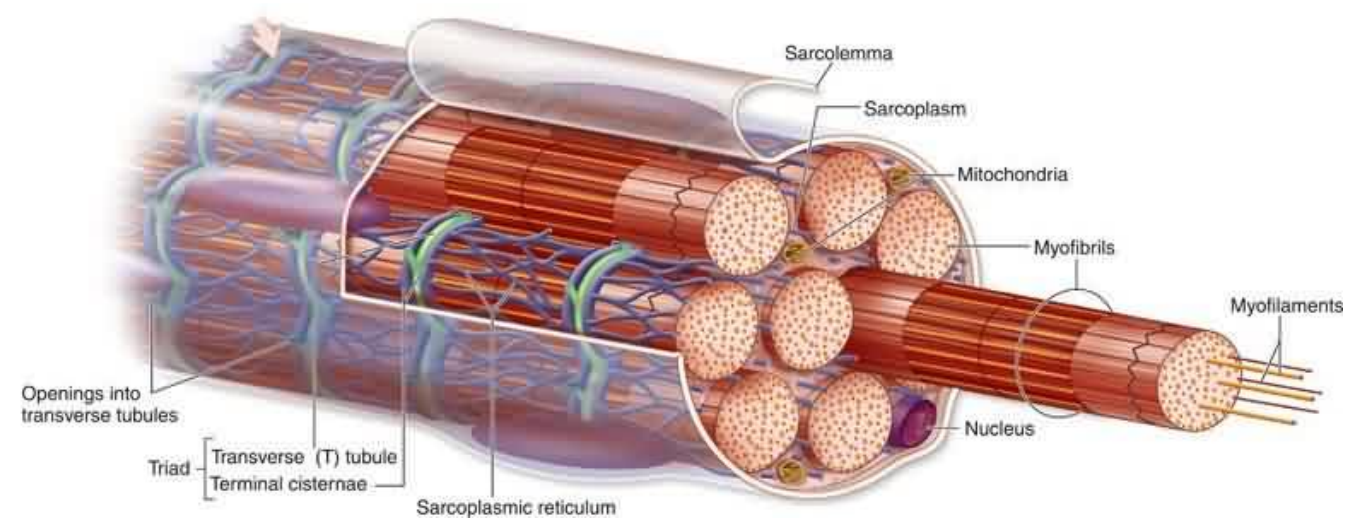

Figure 27: Organization of muscle into complex fibrous groupings [27].

As mentioned earlier, it was found that importing a mesh was not possible, so the built-in COMSOL mesh tool was used to mesh the native STL files. This process in itself was extremely difficult, and resulted in the COMSOL software crashing on numerous occasions. When a mesh was successfully created, it became apparent that the current computer system used to run COMSOL lacked the computational power to run the simulation. 
This challenge made it obvious that model simplification was necessary beyond what was originally anticipated. Since meshing and handling of the geometries by COMSOL was a burden to available computational capabilities, some internal geometries were simplified. The following geometry replacements were made due to mesh difficulties. Instead of accurate curving and branching tubes, the vasculature was simplified to a series of straight cylinders placed in the same locations and angles as the original geometries. Muscles were simplified to be represented by oblong spheres, placed in the same location as original muscle geometries and encompassing the same diameters and lengths. Bone was left in its original form, however the bones of the wrist were excluded due to their small size and compact configuration. Skin was left in its original form.

This simplified model was attempted as a time-dependent simulation, and it was found that the computational time required to successfully run the study would be approximately two months. However, computing the solution as a stationary study produced results within hours. An example of simplified muscle structures can be seen below in Figure 28. 


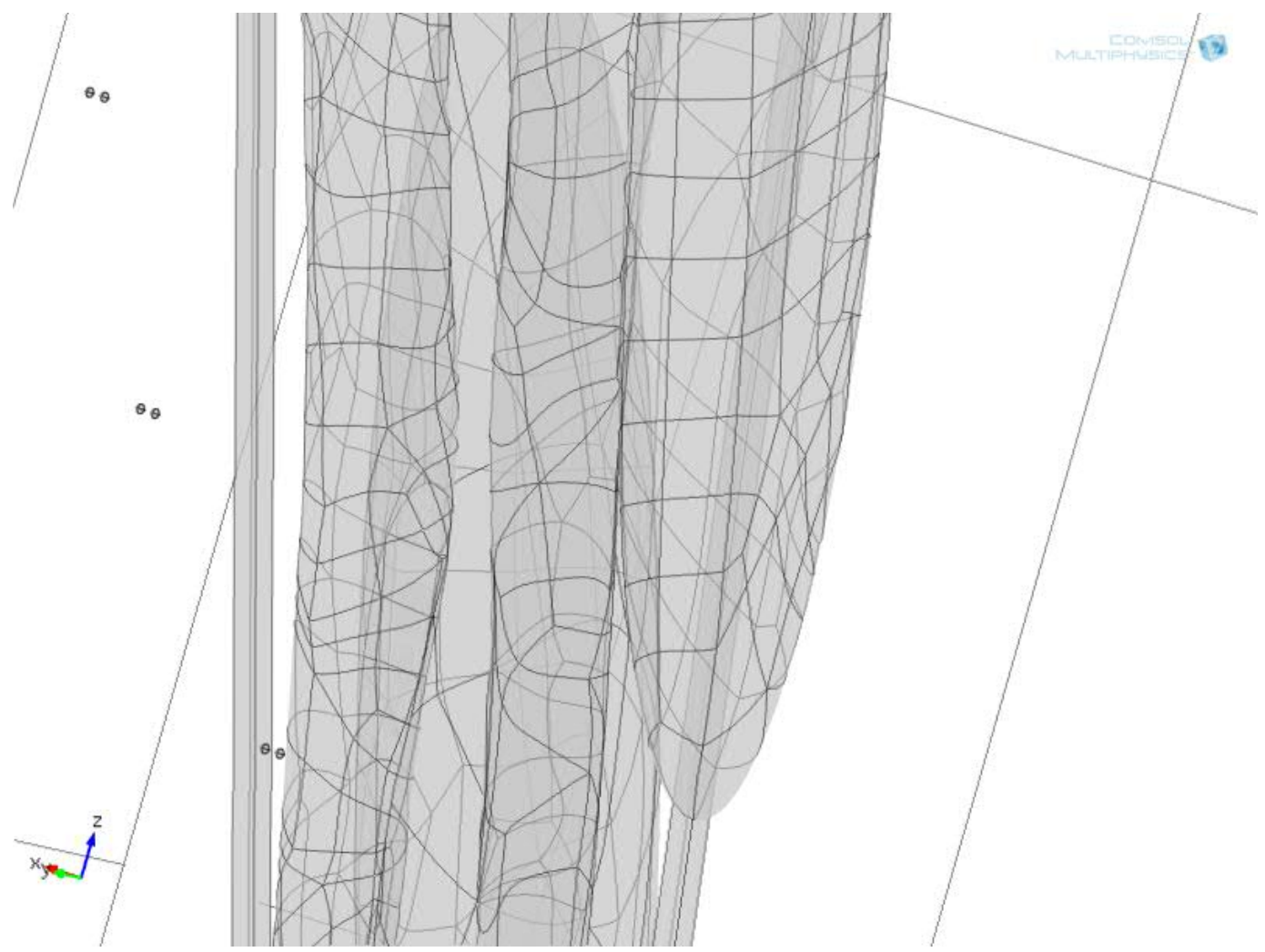

Figure 28: Enlarged view of simplified muscle structures, sectioned from bone to compose the simplified model.

\subsection{Model Validation}

This model was validated by comparison with similar volume field conductor simulations. The primary focus of validation for this study was based the work being developed by Sugden, as described earlier. To determine whether this work resulted in a relevant volume field conductor simulation, material properties were altered to match Sugden's. These properties can be found in Table 2. The properties of materials not present in Sugden's work were adapted from those used in this thesis. Using equivalent material properties, the three dimensional volume conductor field profile developed in this work displayed a similar voltage distribution as the two dimensional volume conductor field profile developed by Sugden. 


\begin{tabular}{lcc}
\hline Tissue & Relative Permittivity, $\varepsilon$ & Electrical Conductivity, $\boldsymbol{\sigma}[\mathbf{S} / \mathbf{m}]$ \\
\hline Muscle & 64.21 & 0.9695 \\
Skin & 42.28 & 0.5495 \\
Bone & 13.77 & 0.1032 \\
Nerve & 35.70 & 0.500 \\
\hline
\end{tabular}

\section{Table 2: Dielectric properties for each tissue used in the validation study and applied in COMSOL.}

These two plots can be seen in Figure 29 and Figure 30 for comparison. The profile developed by Soto as discussed earlier was not used for validation due to the absence of material layers. Sugden's volume conductor plot reaches a maximum electric potential of $0.0668 \mathrm{~V}$ at the surface. The equivalency study performed in this work reached a maximum electric potential of $0.13 \mathrm{~V}$ in the volume. This resulted in a $64 \%$ percent difference between maximum voltages. It was noted in Soto's work that even a small change to the model will greatly affect results. In addition, the incorporation of inhomogeneity into the model increases accuracy of results [17]. Based on these two principles, when adjusting from a simplified two-dimensional model to a complex threedimensional model, a large difference in electric potential can be expected. 


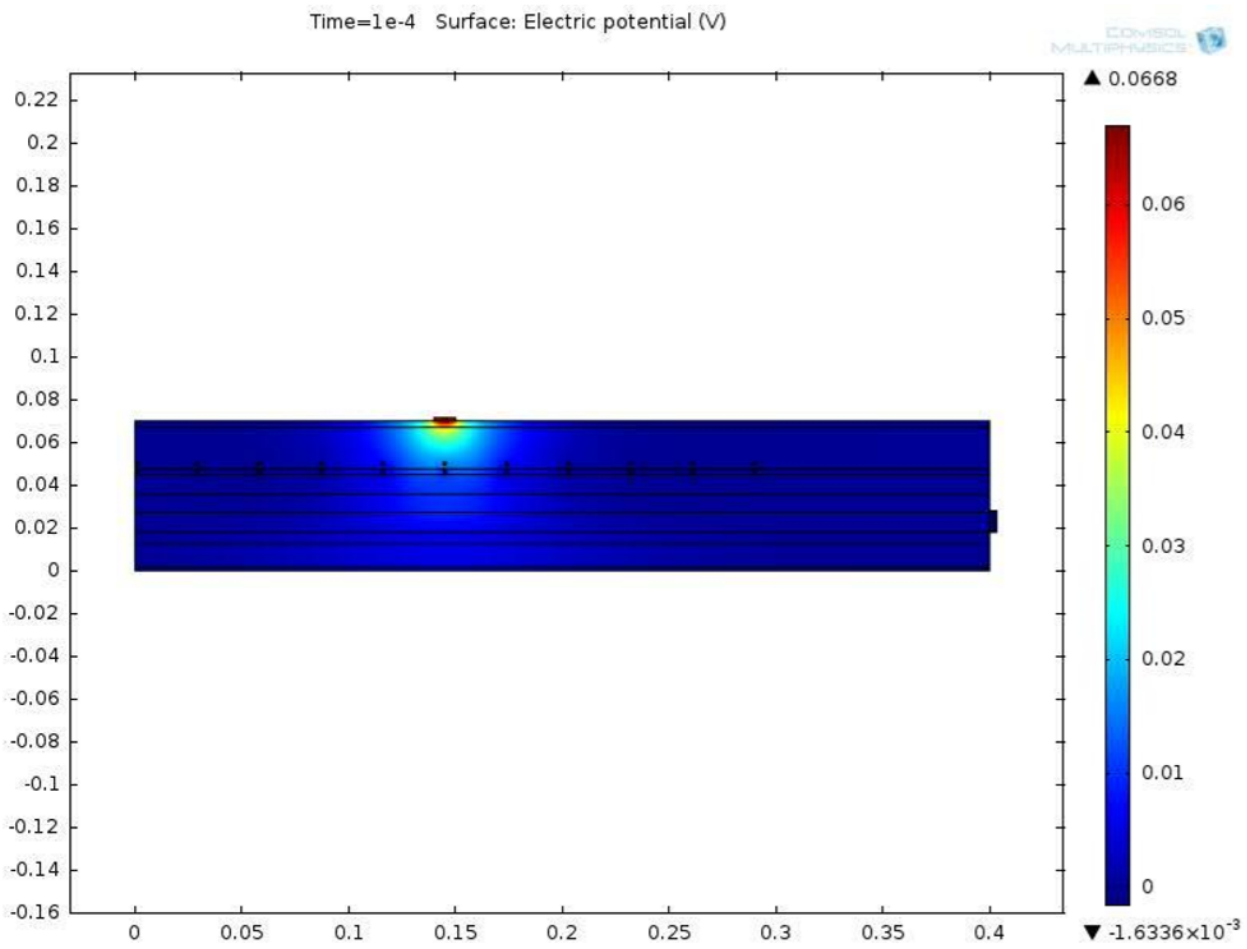

Figure 29: Two-dimensional volume field conductor profile developed by Sugden. 


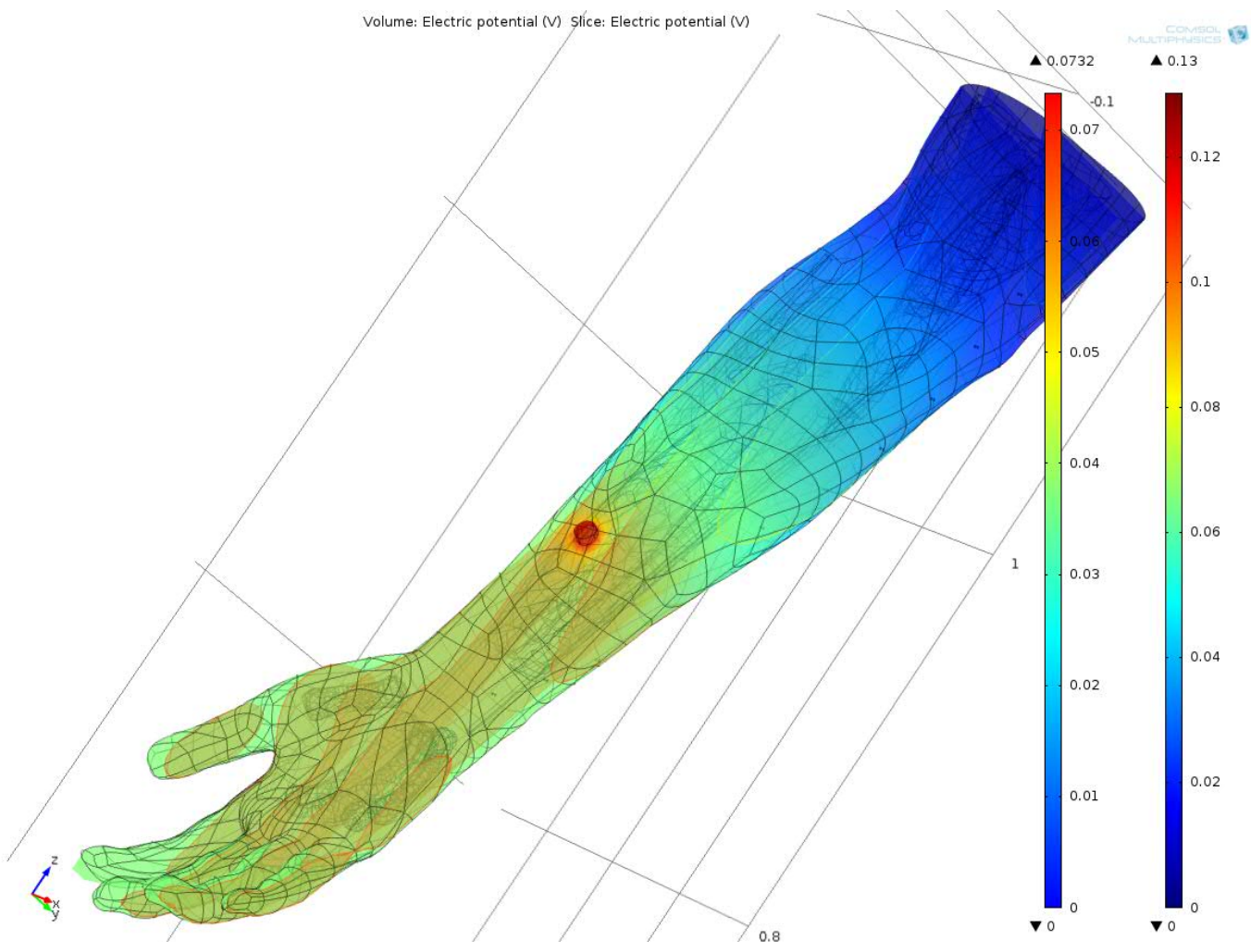

Figure 30: Three-dimensional volume conductor profile developed in this study using equivalent properties for comparison with Figure 29. 


\section{RESULTS}

The study performed in this work yielded significant results. A mesh was successfully developed and converged and volume conductor plots were generated. The following results are presented in this work: mesh convergence, final mesh development, and a volume conductor model representing electric potential in several views performed in a stationary study.

\subsection{Mesh Results and Convergence}

An optimal mesh for the simplified model was found to converge at 390,000 degrees of freedom. Mesh convergence was performed with twelve different meshes, ranging from 90,000 elements to 600,000 elements. Microsoft Excel was used to develop the convergence plot (Figure 31). From this convergence study, a final mesh was settled on consisting of 281,186 elements (Figure 32).

A total of 992 inverted mesh elements were avoided by using linear geometry shape order in the solving process. This feature enables COMSOL to locally fit linear shape functions where higher order shape functions cannot be fitted to the mesh [18]. Resolution is lost locally in these regions; however due to the element size of these inverted elements relative to the entire geometry, it was decided that loss of resolution was an acceptable outcome. The mesh was left with a single inverted mesh element warning at coordinates $(-0.280422,-0.148502,0.707779)$. This inverted mesh element was disregarded due to its location within the geometry. 


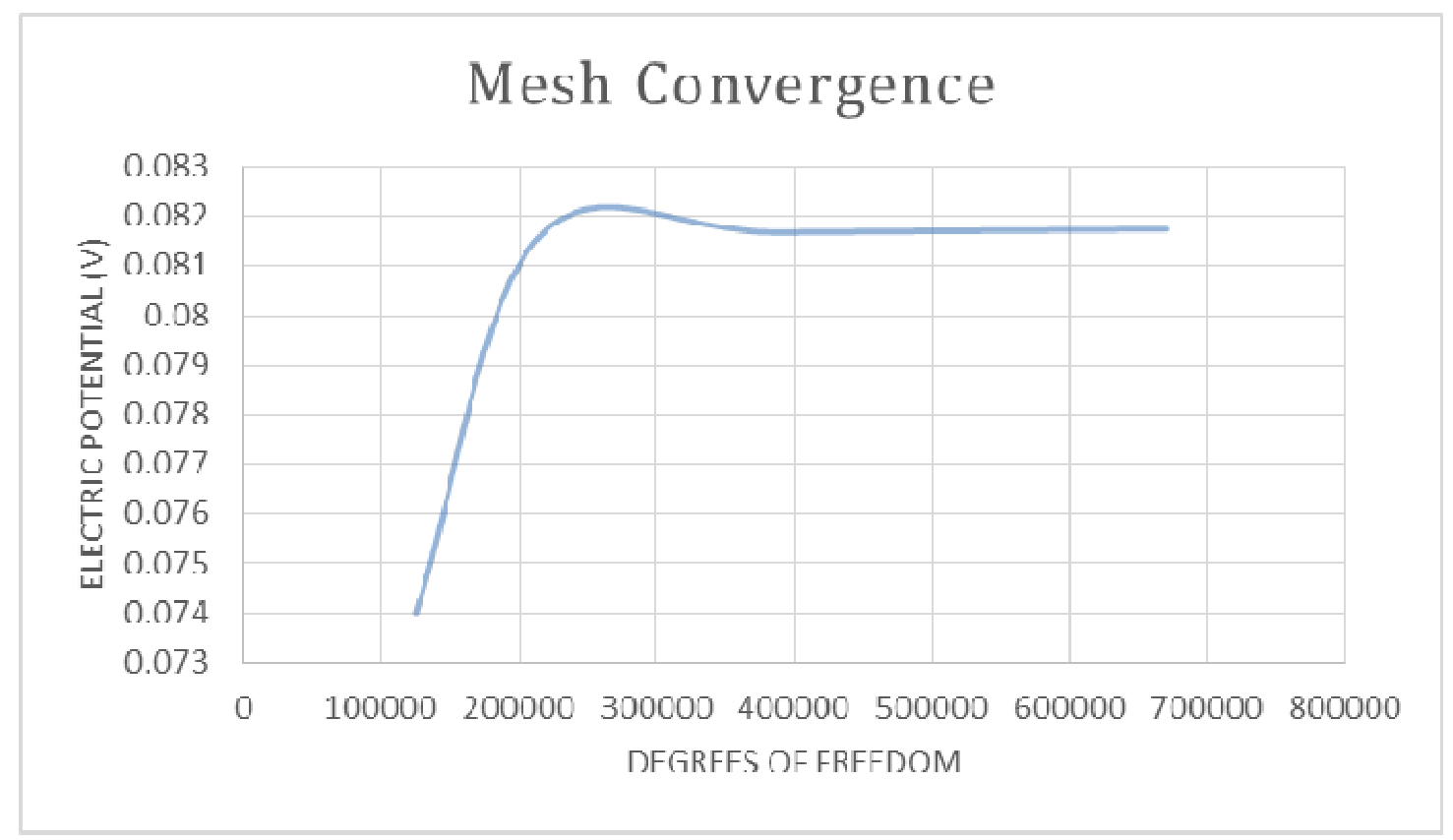

Figure 31: Mesh convergence plot.

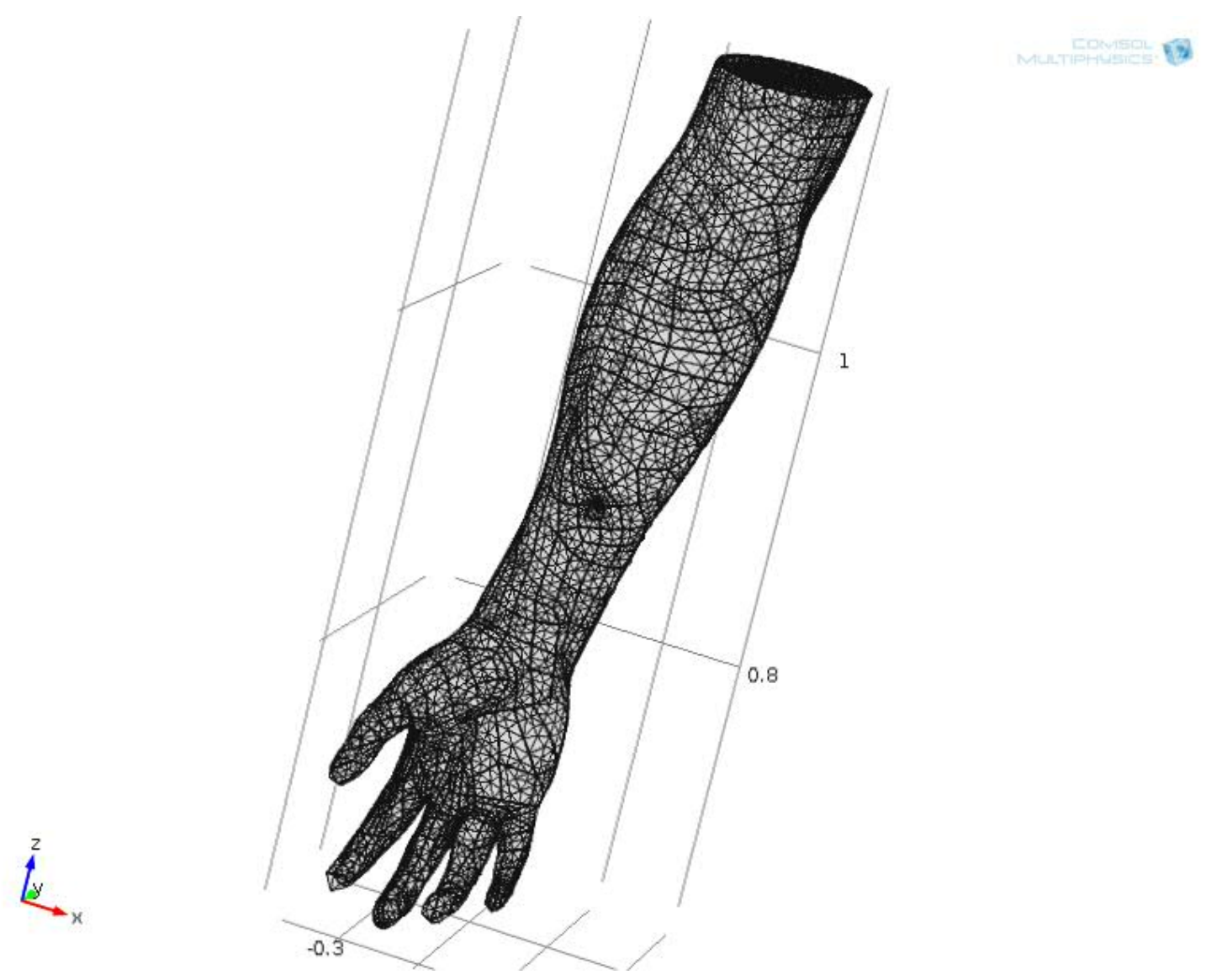

Figure 32: Final mesh used to perform analysis in COMSOL. 
3.2 Simulation Results 
The final study utilized a simplified model subjected to a stationary current source. This simulation solved for 389,534 degrees of freedom using a mesh consisting of 281,186 tetrahedral elements. Greater electric potential was observed in the volume conductor simulation using correct biologic tissue properties than in the validation study. An electric potential surface plot of the entire model with transparent slices for enhanced viewing can be seen in Figure 33. An enlarged view of the electrode interface region can be seen in the upper left corner. The final simulation achieved a maximum electric potential of $0.1645 \mathrm{~V}$. This potential is $26.2 \%$ greater than the maximum voltage achieved in the validation model.

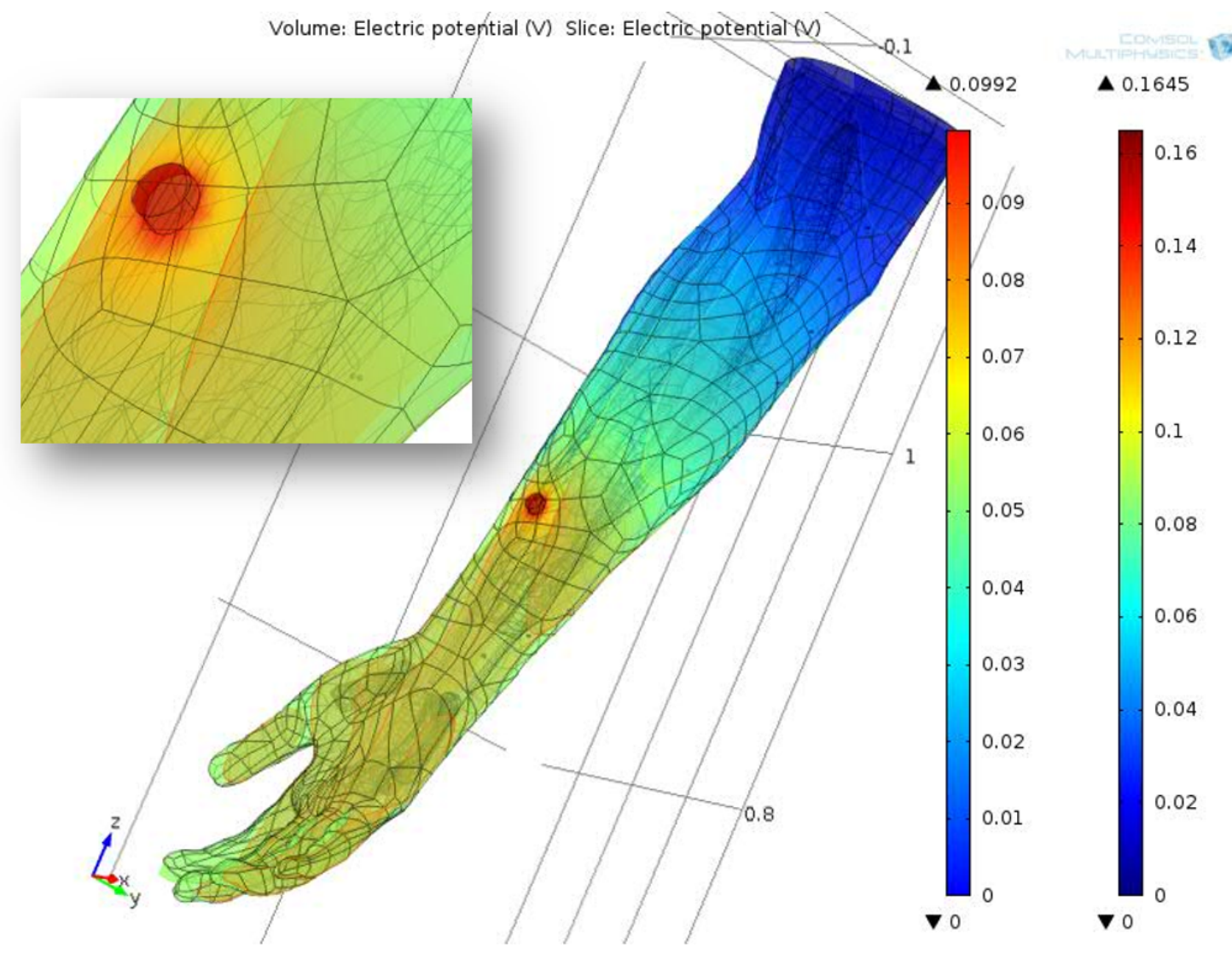

Figure 33: Electric potential plot of the final volume field conductor simplified model. 
For internal, cross-sectional views, cut planes were created to intersect these models. The first cut plane (Figure 34) was used to plot the electric potential trough the forearm, proximal to the electrode and extending to the back of the arm. Figure 35 shows the surface plot of electric potential from this cut plane. Proximal to the electrode, voltage in the tissues reaches up to $0.0955 \mathrm{~V}$. In the distal region of the slice, voltage in the tissues drops to $0.0425 \mathrm{~V}$. The second cut plane (Figure 36) was used to plot the electric potential through a longitudinal cross-section extending on a diagonal through the arm intersecting the electrode. The corresponding surface plot for this plane can be found below in Figure 37. The area surrounding and including the electrode in this slice demonstrates potentials up to $0.1646 \mathrm{~V}$. However, toward the bicep section and where ground was applied, the potential drops to 0V.The third cut plane (Figure 38) was used to plot the cross-section of the forearm, intersecting the electrode. Figure 39 shows the corresponding surface plot of electric potential in this cross-section.

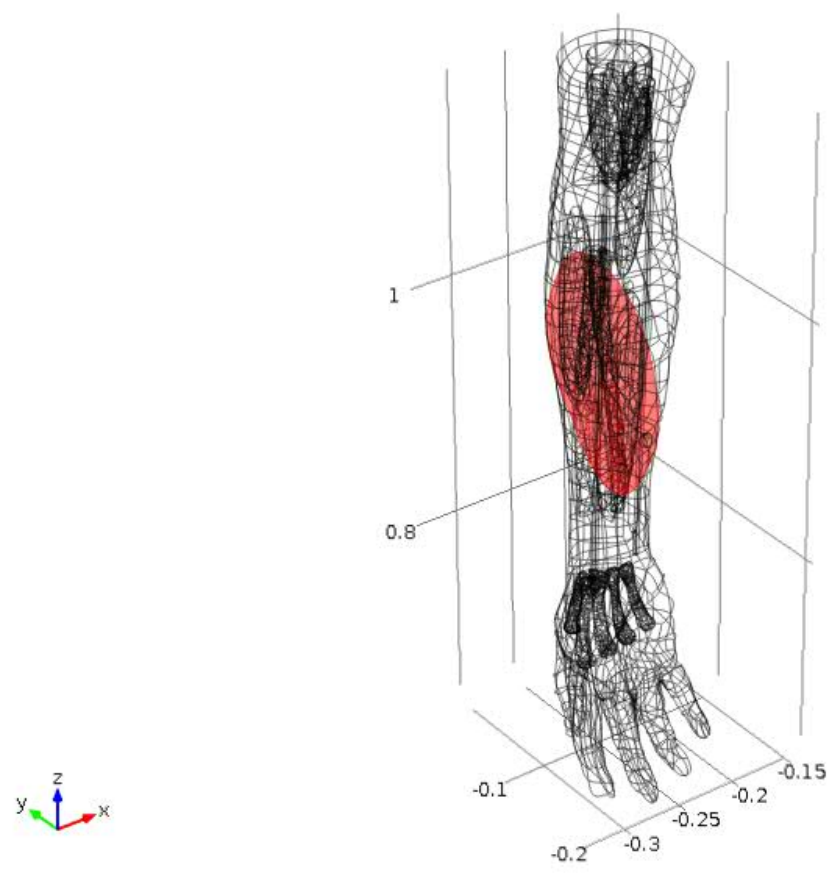


Figure 34: The first cut plane, corresponding to the surface plot of Figure 35.

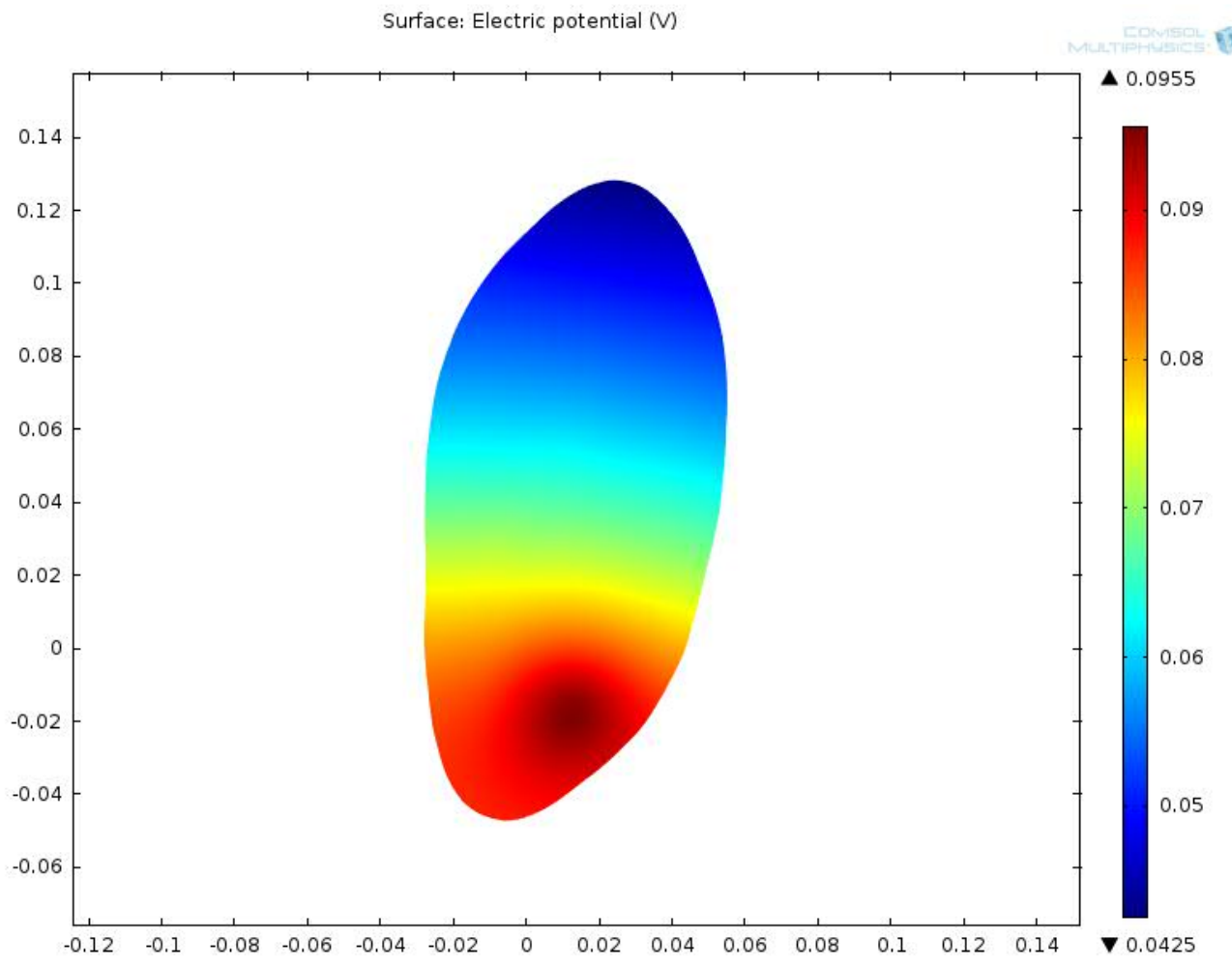

Figure 35: Electric potential surface plot of a slice taken from the volume conductor model. 


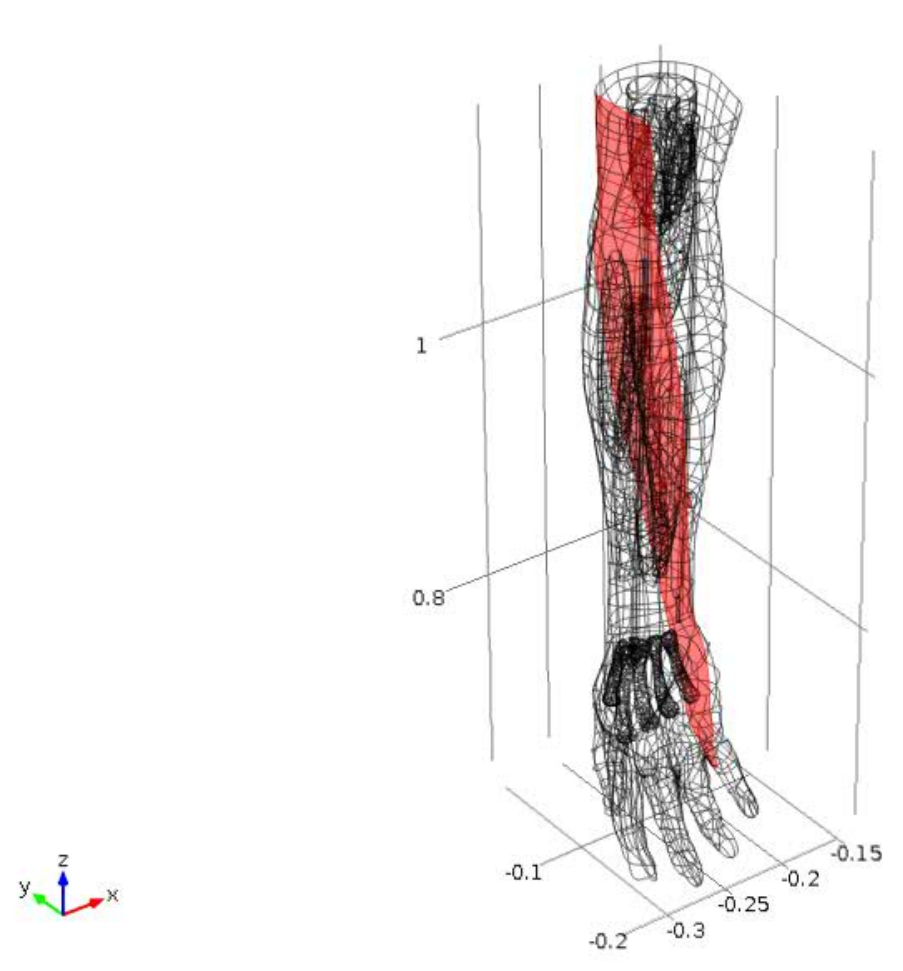

Figure 36: The second cut plane, corresponding to the surface plot of Figure 37. 


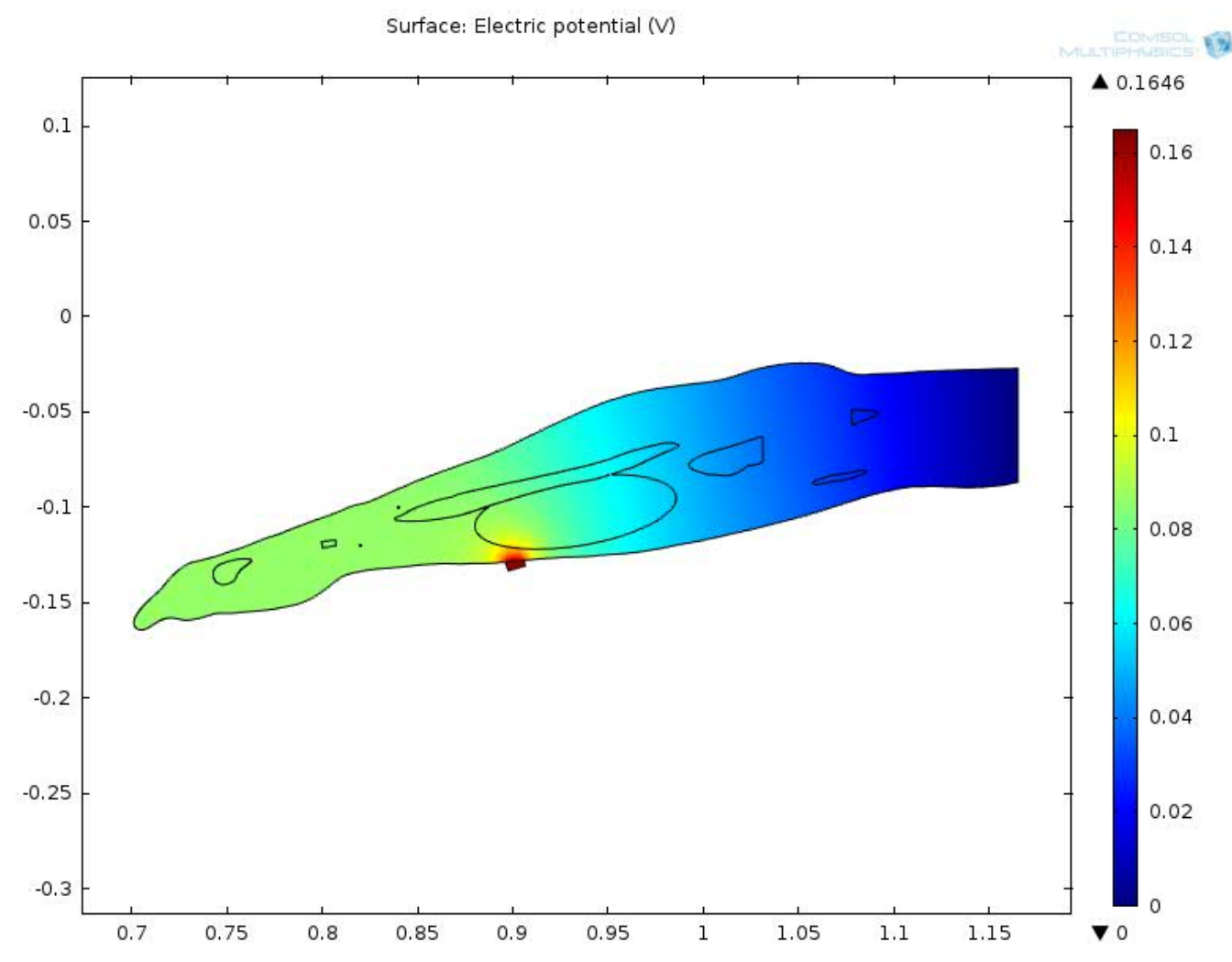

Figure 37: Electric potential surface plot of a slice taken from the volume conductor model. 


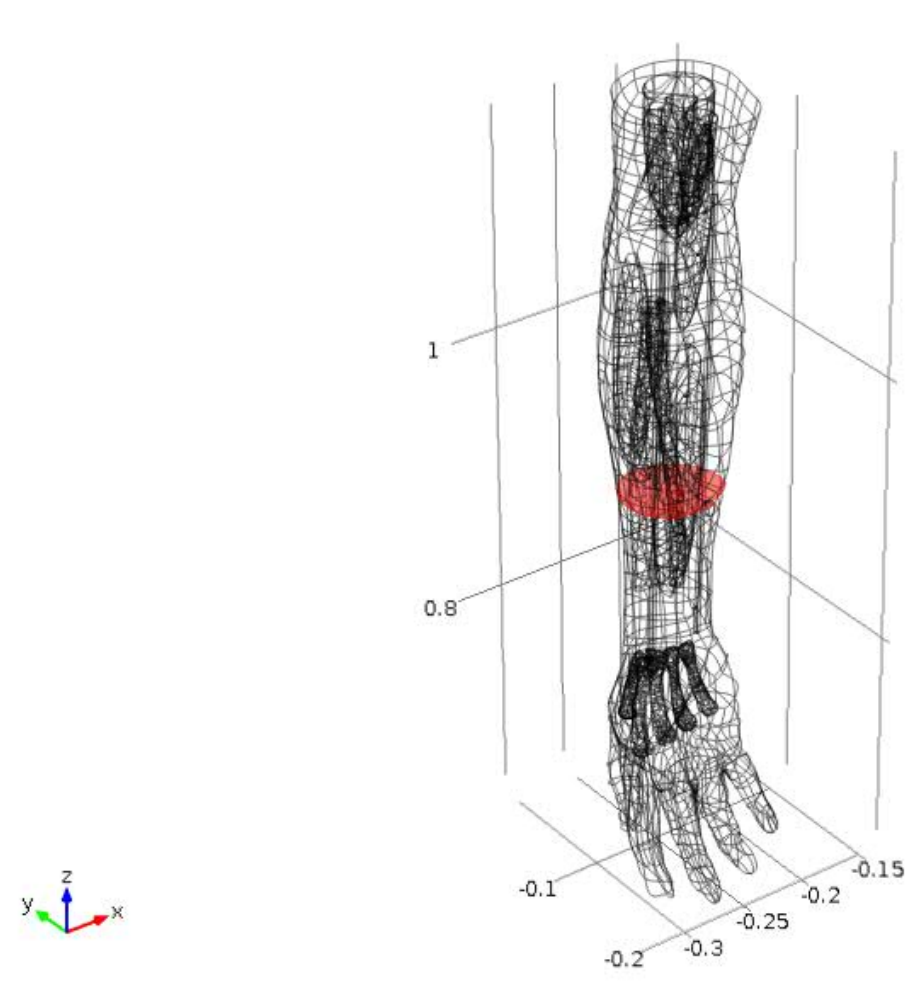

Figure 38: The third cut plane, corresponding to the surface plot of Figure 39. 


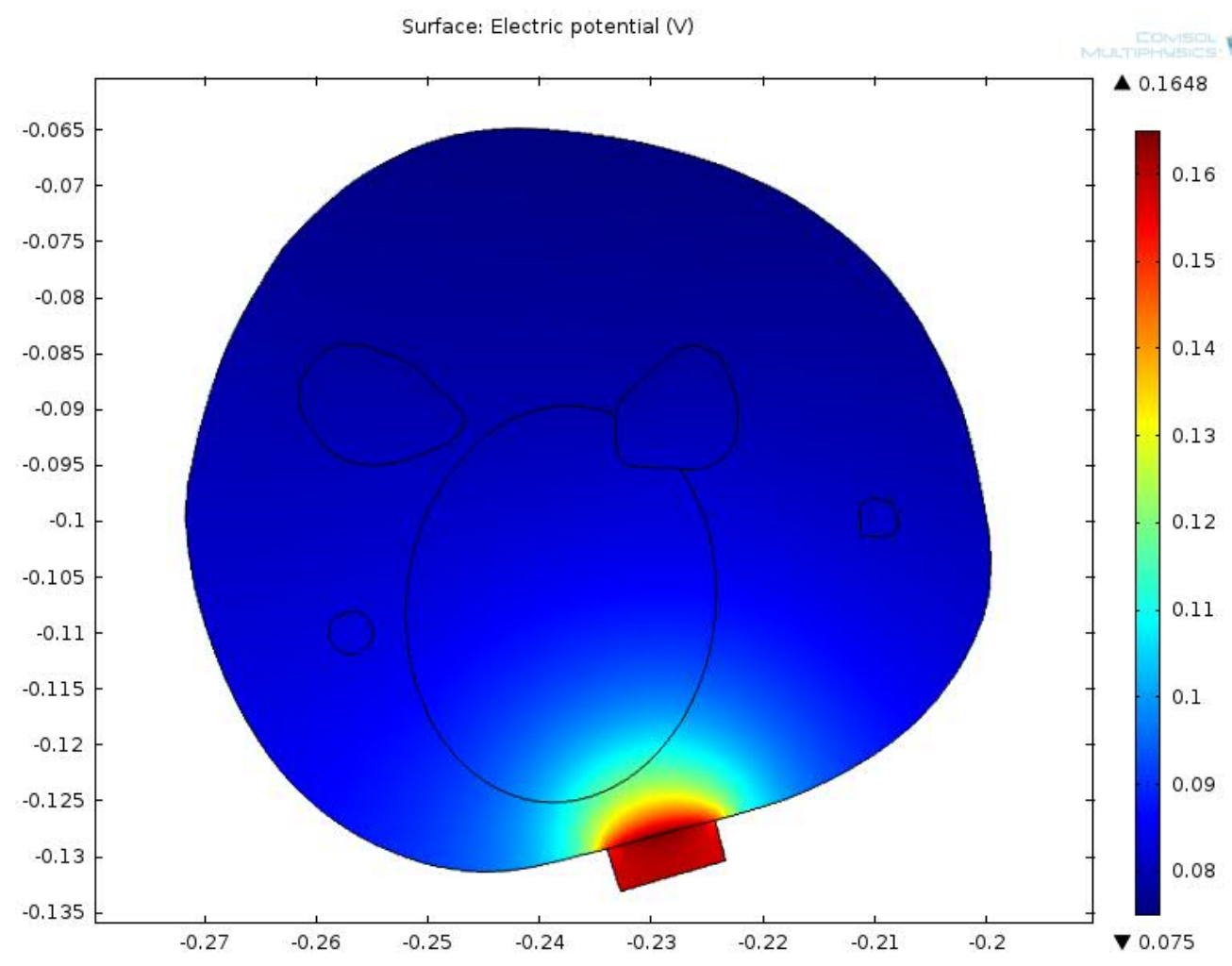

Figure 39: Electric potential surface plot of a slice taken from the volume conductor model.

To determine the effect of material properties on the final solution, two different simulations using the final model were conducted. The first simulation utilized the material properties defined in Table 2 . The second simulation utilized the material properties defined in Table 1. The material property differences between the two models included relative permittivity of skin and nerve and electrical conductivity of muscle and skin. All other material properties were equivalent between the two simulations. The resulting maximum electric potential generated by each simulation was compared.

\subsection{Simulation SPICE Coupling}

Both the final simplified model as well as the complete anatomical model were developed with SPICE simulation coupling in mind. In order to properly import the 
SPICE netlist code into COMSOL, special boundary conditions were included. Each sphere from the assembly of nerve sphere pairs was assigned a "Terminal" boundary condition around its entire surface. The terminal condition provides a connection to the external circuit defined in SPICE. Within the physic module menu, COMSOL contains a SPICE netlist circuitry import option. Once the netlist code is imported into the software, COMSOL converts the circuitry into a series of equivalent terminals and equations. The circuit does not always import successfully, therefore original code must be inspected to ensure that all elements were successfully translated. Missing components must be manually added. 


\section{DISCUSSION}

Previous work in the field of nerve excitation attempted to produce accurate models to describe action potential initiation and human neural recruitment. While these studies were successful in developing models to simulate nerve excitation, they utilized simplified geometrical representations of human tissue. The goal of this work was to develop an anatomically correct, three-dimensional model of the human arm for coupling with SPICE simulations in order to study human neural recruitment and excitation. In this work, a volume conductor field simulation of the human arm was successfully developed. This model incorporated anatomically accurate geometries as well as representative tissue properties.

The anatomical model proved too complex for SPICE coupling in COMSOL at this time and generated the need for a simplified model. The simplified model utilized anatomical structures as well as basic geometries representing structures too complex for meshing. Boundary conditions and nodes applicable to the Electric Currents module were applied. A current source was applied to the skin interface of the electrode. This model was used to successfully generate a volume conductor field simulation.

It was observed in previous studies that a change in material composition of the model can yield large changes in computed potentials. This study provided further evidence that the properties used to represent tissue have a great impact on the simulation. Within this study, two sets of material properties were used: one for validation and another for the final simulation. Adjusting only the dielectric properties described in the material comparison studies resulted in higher electric potentials. 
With the creation of anatomical and simplified three-dimensional structures in this work, overall electric potentials demonstrated significantly greater voltages. Under the assumption that the addition of inhomogeneous material geometries matching anatomical structures improves accuracy, it can be concluded that implementing the full anatomical model defined in this work would produce highly accurate neural recruitment results. This cannot be done until either COMSOL's mesh import capabilities are enhanced or computers with greater computational power become available.

The final simulation conducted in this study utilized stationary solving techniques. Originally the intent of this study was to utilize a time-dependent current source. Due to the limitations of available computational power of the platform used to run COMSOL, a time-dependent simulation was not performed. The advantage of using a time-dependent source is the incorporation of the pulsatile input that mimics the realistic behavior of an electrode. Because this source pulse inputs either $10 \mathrm{~A} / \mathrm{m}^{2}$ or $0 \mathrm{~A} / \mathrm{m}^{2}$, running the stationary model at $10 \mathrm{~A} / \mathrm{m}^{2}$ allowed the volume conductor simulation to be studied as if it were at the peak of an electrode pulse. This approach provides justification for simplifying the study from time-dependent to stationary.

\subsection{Applications}

The final simulation defined in this work can be used with SPICE netlist code to perform nerve fiber activation simulations, and would yield more accurate results than previous studies. Applying simulations to study neuropathies could provide greater understanding into the physiologic causes and effects as well as provide insights into the diagnostics and treatment of those neuropathies. 
This model can also be used in other Biomedical Engineering applications. Other simulations requiring a three-dimensional anatomically accurate model of the arm can utilize the model created in this study and alter the governing module, applicable material properties, and boundary conditions. Examples of such applications include testing of drug delivery devices in-silico and chemical transport through biological tissues.

\subsection{Model Limitations}

Due to limited available computational power, many simplifications had to be made to the fully anatomical model. The original anatomically accurate geometries were replaced with simplified shapes. Fat and cartilage geometries and material properties were excluded from the simulation. Each individual biologic material was assumed to be homogeneous, and intricacies such as the various types of bone and the layers of skin were disregarded. In this study only the partially anatomical model was used to run a simulation due to limited computational power, reducing the accuracy of results. Due to simulation sensitivity to material composition and properties, a relevant difference between the fully anatomical model and the final model in this work would likely be observed.

\subsection{Future Work}

This study illuminated several areas of potential future work. Material properties that are used to represent biologic tissues in a simulation have a significant impact on results. When the material properties in this study were adjusted from the validation simulation to the final simulation, a $23.4 \%$ difference in maximum electrical potential was observed in the results. Previous works have also noted that a small change in the 
properties of material used in simulations will cause a large change in results [17]. With proof that material properties are an integral component for computational models, the need for highly accurate biologic material properties is clear. Future work focusing on this aspect would be a vital contribution to computational modeling in the Biomedical Engineering field. Well controlled in-vivo or in-vitro studies compiled into a database could help accomplish this goal.

Several levels of model simplifications were necessary in this study to perform a simulation with the available computational power. Implementation of the fully anatomical model coupled with SPICE netlist code to model neural membrane electrical characteristics could yield highly accurate neural recruitment results. Soto noted that incorporating inhomogeneous material geometries improves accuracy of results [17]. Based on this statement it can be assumed that implementation of a fully-anatomical model will provide a higher level of accuracy than has previously been achieved. Utilization of a time-dependent study could also improve accuracy of neural recruitment results. A time-dependent current source would implement a pulsed current. The combined effects from multiple pulses could alter the volume conductor profile with time. 


\section{REFERENCES}

[1] J. K. Lindsey and E. N. Marieb, Anatomy \& Physiology, 4th ed. N.p.: Benjamin Cummings, 2011.

[2] E. Chudler. (2009, August). The Brain and Nervous System: Human Diseases and Conditions. [Online]. Available: http://www.humanillnesses.com/BehavioralHealth-A-Br/The-Brain-and-Nervous-System.html

[3] L. Zhou and S. Y. Chiu, "Computer model for action potential propagation through branch point in myelinated nerves," Journal of neurophysiology, vol. 85, no. 1, pp. 197-210, 2001.

[4] OpenStax College. (2013, June 28). The Action Potential [Online]. Available: http://cnx.org/content/m46526/1.4/

[5] J. Kimball. (2013, May 6). Synapses [Online]. Available: http://users.rcn.com/jkimball.ma.ultranet/BiologyPages/S/Synapses.html

[6] B. Nazario. (2014, January 16). Early Symptoms of Multiple Sclerosis (MS): Tingling, Numbness, Balance, and More [Online]. Available: http://www.webmd.com/multiple-sclerosis/guide/multiple-sclerosisdiagnosis?page $=2$

[7] M.K. Houtchens, "Multiple sclerosis and other inflammatory demyelinating diseases of the central nervous system," Bradley's Neurology in Clinical Practice, 6th ed. Philadelphia, PA: Elsevier Saunders, 2012.

[8] NINDS. (2014, April 16). National Institute of Neurological Disorders and Stroke: Peripheral Neuropathy Fact Sheet [Online]. Available: http://www.ninds.nih.gov/disorders/peripheralneuropathy/detail_peripheralneurop athy.htm

[9] NIH. (2014). Degenerative Nerve Diseases: MedlinePlus. U.S. National Library of Medicine [Online]. Available:

http://www.nlm.nih.gov/medlineplus/degenerativenervediseases.html

[10] EMB. IEEE Engineering in Medicine \& Biology Society: Neural Engineering. [Online]. Available: http://www.embs.org/about-biomedical-engineering/ourareas-of-research/neural-engineering

[11] NIBIB. (2014) National Institute of Biomedical Imaging and Bioengineering: Computational Modeling [Online]. Available: http://www.nibib.nih.gov/scienceeducation/science-topics/computational-modeling 
[12] S. Hazelwood. Class Lecture. Applied Finite Element Analysis. California Polytechnic State University, San Luis Obispo, CA. date, September, 2014.

[13] U.S. Food and Drug Administration. Reporting of Computational Modeling Studies in Medical Device Submissions: Draft Guidance for Industry and Food and Drug Administration Staff. [Online]. Available:

http://www.fda.gov/MedicalDevices/DeviceRegulationandGuidance/GuidanceDo cuments/ucm371016.htm

[14] COMSOL Multiphysics. Multiphysics Software Product Suite. [Online]. Available: http://www.comsol.com/products

[15] G. Bishop, "The relation between nerve fiber size and sensory modality: phylogenetic implications of the afferent innervation of cortex," The Journal of nervous and mental disease, vol. 128, pp 89-114, 1959

[16] R.B. Szlavik, and H. de Bruin, "The effect of stimulus current pulse width on nerve fiber size recruitment patterns," Medical Engineering \& Physics, vol. 21, pp. 507$515,1999$.

[17] N. D. Soto, "Characterizing Nerve Fiber Activation by Varying Fiber Diameter and Depth Within a Conductive Medium: A Finite Element Approach," M.S. thesis, Dept. Biomed. Eng., California Polytechnic State Univ., San Luis Obispo, CA, 2011.

[18] I. Kjerberg. (2012, January 31). COMSOL Discussion Forum: Geometry Solving. [Online]. Available: http://www.comsol.com/community/forums/general/thread/25753

[19] Cadence Design Systems. (2014, June 10). OrCAD EE (PSpice) Designer Overview. [Online]. Available: http://www.orcad.com/products/orcad-ee-designer/overview

[20] N.K. Mitsuhashi, T. Fujieda, S. Tamura, T. Kawamoto, Takagi, and K. Okubo., "BodyParts3D: 3D Structure Database for Anatomical Concepts," Nucleic Acids Research, vol 37 pp. 782-785, 2009.

[21] R. Scopigno. MeshLab: Visual Computing Laboratory [Online]. Available: http://www.meshlab.org

[22] C. Gabriel, S. Gabriel and E. Corthout, "The dielectric properties of biological tissues: I. Literature survey,” Phys. Med. Biol., vol. 41, pp. 2231-2249, 1996.

[23] D. Miklavčič, N. Pavšelj, and F. X. Hart, "Electric properties of tissues," Wiley encyclopedia of biomedical engineering, 2006). 
[24] L.A. Geddes, and L.E. Baker. Principles of Applied Biomedical Instrumentation. $3^{\text {rd }}$ ed. New York: Wiley, 1968.

[25] C. Furse, A. Douglas and C. H. Durney, Basic Introduction to Bioelectromagnetics. CRC Press, 2009.

[26] R.B. Szlavik and G. E. Turner, "A novel method for characterization of peripheral nerve fiber size distributions by group delay measurements and simulated annealing optimization," Annual International Conference of the IEEE Engineering in Medicine and Biology Society: IEEE Engineering in Medicine and Biology Society, 2008.

[27] A. Kats. (2012) Anatomy and Physiology: Muscles. [Online.] Available: http\%3A\%2F\%2Ffau.pearlashes.com\%2Fanatomy\%2FChapter\%252014\%2FCha pter\%252014.htm 